This item was submitted to Loughborough's Research Repository by the author.

Items in Figshare are protected by copyright, with all rights reserved, unless otherwise indicated.

\title{
The Schur decomposition of the velocity gradient tensor for turbulent flows
}

PLEASE CITE THE PUBLISHED VERSION

https://doi.org/10.1017/jfm.2018.344

PUBLISHER

(c) Cambridge University Press

VERSION

AM (Accepted Manuscript)

LICENCE

CC BY-NC-ND 4.0

REPOSITORY RECORD

Keylock, Chris. 2019. "The Schur Decomposition of the Velocity Gradient Tensor for Turbulent Flows". figshare. https://hdl.handle.net/2134/33700. 


\title{
The Schur decomposition of the velocity gradient tensor for turbulent flows
}

\author{
Christopher J. Keylock ${ }^{1} \dagger$ \\ ${ }^{1}$ School of Architecture, Building, and Civil Engineering, Loughborough University, \\ Loughborough, Leicestershire, LE11 3TU, U.K.
}

(Received ?; revised ?; accepted ?. - To be entered by editorial office)

The velocity gradient tensor for turbulent flow contains crucial information on the topology of turbulence, vortex stretching and the dissipation of energy. A Schur decomposition of the velocity gradient tensor (VGT) is introduced to supplement the standard decomposition into rotation and strain tensors. Thus, the normal parts of the tensor (represented by the eigenvalues) are separated explicitly from non-normality. Using a direct numerical simulation of HIT, it is shown that the norm of the non-normal part of the tensor is of a similar magnitude to the normal part. It is common to examine the second and third invariants of the characteristic equation of the tensor simultaneously (the $\mathrm{Q}-\mathrm{R}$ diagram). With the Schur approach, the discriminant function separating real and complex eigenvalues of the VGT has an explicit form in terms of strain and enstrophy: where eigenvalues are all real, enstrophy arises from the non-normal term only. Re-deriving the evolution equations for enstrophy and total strain highlights the production of nonnormality and interaction production (normal straining of non-normality). These cancel when considering the evolution of the VGT in terms of its eigenvalues but are important for the full dynamics. Their properties as a function of location in $\mathrm{Q}-\mathrm{R}$ space are characterized. The Schur framework is then used to explain two properties of the VGT: the preference to form disc-like rather than rod-like flow structures, and the vorticity vector and strain alignments. In both cases, non-normality is critical for explaining behaviour in vortical regions.

Key words:

\section{Introduction}

\subsection{Brief overview}

This paper is concerned with properties of the velocity gradient tensor (VGT) for incompressible, homogeneous, isotropic turbulence (HIT). This is a classical topic in turbulence fluid mechanics as HIT is the testing ground for a great deal of turbulence theory as the equations for the evolution for the VGT are derived by taking the spatial gradient of the Navier-Stokes equations. Hence, as studies of the VGT refine knowledge of flow structure topologies, distortion and their interaction with dissipation (Tsinober et al. 1997), the physical understanding of the cascades of energy and enstrophy are enhanced, leading to new conceptual models (Lundgren 1982; Goto 2008) and better prospects for practical modelling of turbulent flows. A recent review paper by Meneveau (2011) provides a great deal of information on the properties of the VGT and serves as both a basis

$\dagger$ Email address for correspondence: c.j.keylock@lboro.ac.uk 
and a point of departure for this study. The key difference concerns the form of decomposition of the tensor. Conventional studies employ the clearly physically interpretable Hermitian/skew-Hermitian decomposition into strain, S, and rotation, $\Omega$, components, with the latter often then expressed as a vorticity vector. However, the eigenvalues of the VGT are also important for delimiting different topological states of the tensor, and therefore there is some history to studying the invariants of the characteristic equation for the VGT (Kerr 1985; Ashurst et al. 1987; Cantwell 1993; Jeong \& Girimaji 2003; Elsinga \& Marusic 2010; Paul et al. 2017), as well as modelling their evolution (Girimaji \& Pope 1990; Cantwell 1992; Martin et al. 1998; Nomura \& Post 1998; Biferale et al. 2007; Li \& Meneveau 2007). The approach proposed here attempts to unify both eigenvalue and strain/rotation frameworks and, as a consequence, helps elucidate important aspects of the flow physics.

The first thing noted is that just examining the eigenvalues is insufficient for developing an alternative decomposition of the VGT. While the Hermitian/skew-Hermitian decomposition gives an additive decomposition of the velocity gradient tensor, $\mathrm{A}$ :

$$
\mathrm{A}=\mathrm{S}+\Omega
$$

to form the equivalent with an eigenvalue-based approach, a decomposition of the tensor into a normal tensor, B, (characterized by the eigenvalues) and a non-normal part, C, (characterizing the tensor asymmetries) is needed. Hence, an alternative to (1.1) may be written as

$$
A=B+C
$$

The tool from matrix algebra used to accomplish this is the Schur transform (Schur 1909). Having commenced the analysis of A from this starting point, the Hermitian/skewHermitian decompositions of $B$ and $C$ can be used to elucidate the relative importance of strain and rotation for both the normal and non-normal parts of the tensor. This framework may then be employed to re-interrogate and shed further light on a number of properties of the VGT.

The plan of this manuscript is to review key mathematical and physical aspects of the velocity gradient tensor in the remainder of the introduction, establishing concepts to be used as the basis or point-of-departure for the Schur approach. In Section 2 we then introduce the Schur decomposition and use it to derive the decomposition in (1.2). Taking the strain and rotation components of these tensors permits the core concepts from the introduction to be stated in a new way (Sections 2.2 and 2.3), as well as to derive the evolution equations for strain and rotation in this framework (Section 2.4). The final two parts of Section 2 use the derived terms to formulate metrics to characterize the physical aspects of the tensor that are employed in the results sections. The first results, in Section 4, concern the importance of non-normality for the tensor in terms of norms, enstrophy, strain and the respective production terms. Section 5 then examines the strain rate tensor to show how turbulence forms disc-like structures. Finally, Section 6 reinterrogates vorticity vector and strain eigenvector alignments to explain the preferential alignment between the vorticity vector and the eigenvector for the intermediate strain eigenvalue.

\subsection{The velocity gradient tensor and the invariants of its characteristic equation}

The velocity gradient tensor, $\mathrm{A} \in \Re^{3 \times 3}$, is given by $A_{i j}=\partial u_{i} / \partial x_{j}$, where $u$ is a velocity component and $x$ is a spatial direction. It is directly related to the Navier-Stokes 
equations,

$$
\frac{\partial}{\partial t} \mathbf{u}+\mathbf{u} \cdot \nabla \mathbf{u}=-\frac{1}{\rho} \nabla p+\nu \Delta \mathbf{u}
$$

where $t$ is time, $p$ is the pressure, $\rho$ is the density and $\nu$ is the kinematic viscosity. This may be made explicit by taking the spatial gradient of the Navier-Stokes equations:

$$
\frac{\partial}{\partial t} \mathrm{~A}+\mathbf{u} \cdot \nabla \mathrm{A}=-\mathrm{A}^{2}-\mathrm{H}+\nu \Delta \mathrm{A}
$$

where $\mathrm{H}$ is the Hessian of the kinematic pressure field, i.e. $H_{i j}=\frac{\partial^{2} p}{\partial x_{i} \partial x_{j}}$. The characteristic equation for $A$ is

$$
\lambda_{i}^{3}+\mathrm{P}_{A} \lambda_{i}^{2}+\mathrm{Q}_{A} \lambda_{i}+\mathrm{R}_{A}=0
$$

where the $\lambda_{i}$ are the eigenvalues of the tensor. Clearly, the three invariants of this equation may be expressed in terms of the eigenvalues and for an incompressible flow, because there is zero trace, $\operatorname{tr}(\mathrm{A})=0$, and $\mathrm{P}_{A}=\sum \lambda_{i}$, it follows that $\mathrm{P}_{A}=0$. The second and third invariants, $\mathrm{Q}_{A}$ and $\mathrm{R}_{A}$ are given by:

$$
\begin{aligned}
& \mathrm{Q}_{A}=-\frac{1}{2} \operatorname{tr}\left(\mathrm{A}^{2}\right) \equiv\left(1-\delta_{i j}\right) \sum \lambda_{i} \lambda_{j} \\
& \mathrm{R}_{A}=-\operatorname{det}(\mathrm{A}) \equiv \prod \lambda_{i}
\end{aligned}
$$

where $\delta_{i j}$ is the Kronecker delta. These expressions for $\mathrm{Q}_{A}$ and $\mathrm{R}_{A}$ are of significance topologically, because the sign of the discriminant function for incompressible flow

$$
\Delta_{L}=\mathrm{Q}_{A}^{3}+\frac{27}{4} \mathrm{R}_{A}^{2}
$$

separates regions where the eigenvalues form a conjugate pair $\left(\Delta_{L}>0\right)$ and where they are all real $\left(\Delta_{L}<0\right)$. In the Lagrangian frame of a moving fluid element, the former results in closed streamlines, explaining the use of $\Delta_{L}>0$ as a local, practical tool for coherent structure identification (Chong et al. 1990), although because $\mathrm{Q}_{A}$ is raised to an odd power in (1.8), it follows that $\mathrm{Q}_{A}>0$ is a more restrictive definition (Hunt et al. 1988; Dubief \& Delcayre 2000). Because of this physical meaning to the change in eigenvalue behaviour, it follows that particular regions of joint $\mathrm{Q}_{A}-\mathrm{R}_{A}$ space have topological interpretation (Perry \& Chong 1987):

- $\Delta_{L}>0, \mathrm{R}_{A}>0$ - compressing of the flow towards an unstable focus region;

- $\Delta_{L}>0, \mathrm{R}_{A}<0$ - stretching of the flow away from a stable focus region;

- $\Delta_{L}<0, \mathrm{R}_{A}>0$ - two saddles with an unstable node;

- $\Delta_{L}<0, \mathrm{R}_{A}<0$ - two saddles with a stable node.

Alternatively, $\mathrm{Q}_{A}$ and $\mathrm{R}_{A}$ can be defined in terms of strain and rotation tensors. $\mathrm{A}$ Hermitian-skew Hermitian decomposition into strain and rotation is given by

$$
\begin{aligned}
S_{A} & =\frac{1}{2}\left(A+A^{*}\right) \\
\Omega_{A} & =\frac{1}{2}\left(A-A^{*}\right)
\end{aligned}
$$




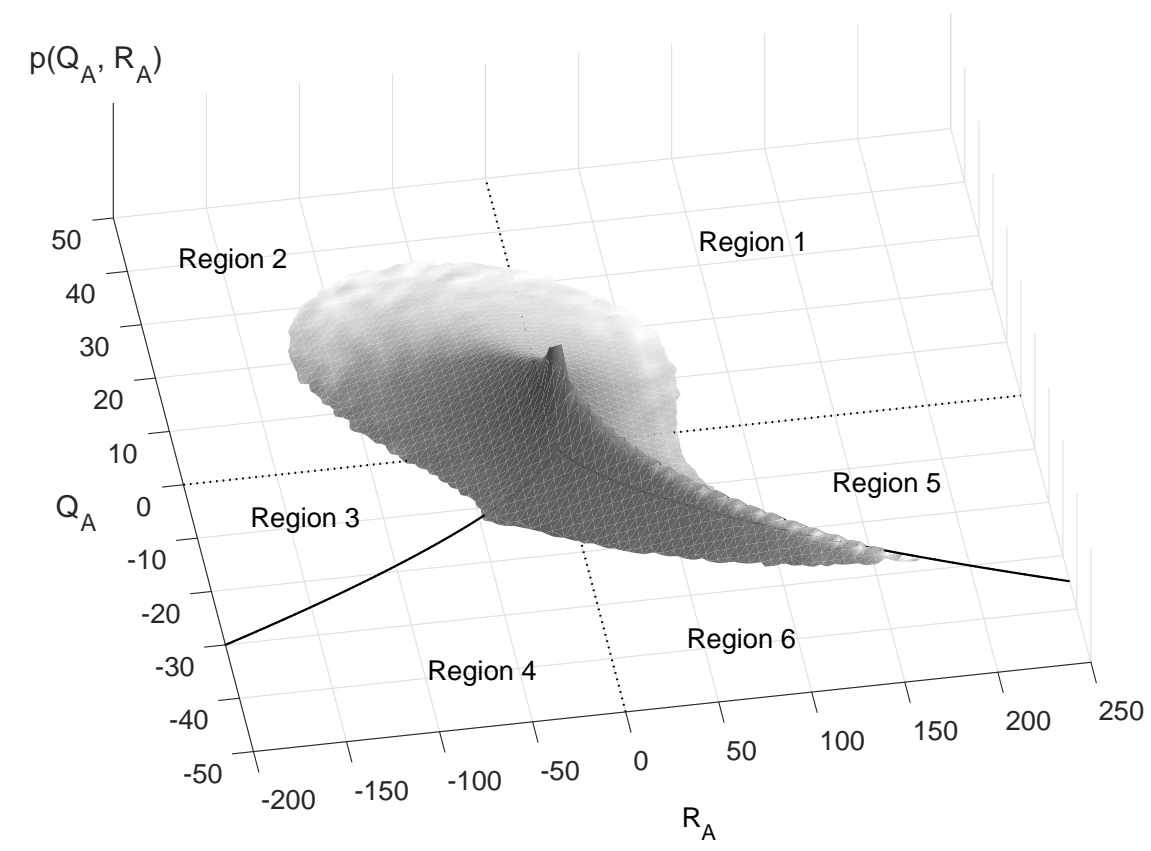

Figure 1. A schematic of the joint distribution function that forms the $\mathrm{Q}_{A}-\mathrm{R}_{A}$ diagram, plotted as a surface, with the discriminant function, $\Delta_{L}=0$ shown as a solid line and the six regions used throughout this study highlighted. The Vieillefosse tail is the elongated feature between regions 5 and 6 close to where $\Delta_{L}=0$ (Vieillefosse 1984). This figure was constructed using the results stored in the Johns Hopkins database (Li et al. 2008).

where the $*$ superscript is the conjugate transpose. This leads to

$$
\begin{aligned}
\mathrm{Q}_{A} & =\frac{1}{2}\left(\left\|\Omega_{A}\right\|^{2}-\left\|\mathrm{S}_{A}\right\|^{2}\right) \\
& \equiv \mathrm{Q}_{A}^{(\Omega)}-\mathrm{Q}_{A}^{(S)} \\
\mathrm{R}_{A} & =-\operatorname{det}\left(\mathrm{S}_{A}\right)-\operatorname{tr}\left(\Omega_{A}^{2} \mathrm{~S}_{A}\right) \\
& \equiv \mathrm{R}_{A}^{(S)}-\operatorname{tr}\left(\Omega_{A}^{2} \mathrm{~S}_{A}\right),
\end{aligned}
$$

where, e.g. $\left\|S_{A}\right\|=\sqrt{\operatorname{tr}\left(S_{A} S_{A}^{*}\right)}$ is the Frobenius norm and one may also choose to work with the vorticity vector, $\boldsymbol{\omega}$, where each element is

$$
\omega_{i}=-\epsilon_{i j k} \Omega_{j k}
$$

and $\epsilon_{i j k}$ is the Levi-Cevita symbol. Equations (1.11) and (1.12) give physical interpretations of the invariants as the excess of enstrophy with respect to total strain and the excess of strain production with respect to enstrophy production, respectively. It should be noted that while $\mathrm{Q}_{A}$ is therefore the difference between two positive quantities, $\mathrm{R}_{A}$ reflects a balance between two terms, for which both mean values are positive (Taylor 1938; Betchov 1956), but the instantaneous values may take either sign. This provided the motivation for Lüthi and co-workers to examine the velocity gradient tensor from the perspective of a $\mathrm{Q}_{A} ;-\operatorname{det}\left(\mathrm{S}_{A}\right) ; \operatorname{tr}\left(\Omega_{A}^{2} \mathrm{~S}_{A}\right)$ decomposition (Lüthi et al. 2009).

The well-known $\mathrm{Q}-\mathrm{R}$ diagram $\left(\mathrm{Q}_{A}-\mathrm{R}_{A}\right.$ in this paper's notation) is shown for 
TABLE 1. Occupancy (expressed as a \% of realizations) of different states for HIT for the velocity gradient tensor, A. For compactness, the $A$ subscript is not used in the table headings. Hence, $\mathrm{R}^{(S)} \equiv-\operatorname{det}\left(\mathrm{S}_{A}\right)$ and $\Omega^{2} S \equiv \operatorname{tr}\left(\Omega_{A}^{2} \mathrm{~S}_{A}\right)$.

\begin{tabular}{|c|c|c|c|c|c|c|}
\hline Regio & & $\begin{array}{l}\mathrm{R}^{(S)}>0 \\
\Omega^{2} S>0\end{array}$ & $\begin{array}{c}\mathrm{R}^{(S)}>0, \\
\Omega^{2} S<0\end{array}$ & $\begin{array}{l}\mathrm{R}^{(S)}< \\
\Omega^{2} S<\end{array}$ & $\begin{array}{c}\mathrm{R}^{(S)}<0, \\
\Omega^{2} S>0\end{array}$ & Total \\
\hline 1 & $Q_{A}>0, R_{A}>0$ & 1.8 & 4.0 & 5.3 & 0 & 11.1 \\
\hline 2 & $Q_{A}>0, R_{A}<0$ & 20.0 & 0 & 0.8 & 5.7 & 26.5 \\
\hline 3 & $Q_{A}<0, \Delta_{L}>0, R_{A}<0$ & 5.3 & 0 & 1.0 & 3.6 & 9.9 \\
\hline 4 & $Q_{A}<0, \Delta_{L}<0, R_{A}<0$ & 4.0 & 0 & 3.0 & 2.0 & 9.0 \\
\hline 5 & $Q_{A}<0, \Delta_{L}>0, R_{A}>0$ & 7.0 & 5.0 & 1.0 & 0 & 13.0 \\
\hline 6 & $Q_{A}<0, \Delta_{L}<0, R_{A}>0$ & 26.2 & 3.3 & 1.0 & 0 & 30.5 \\
\hline
\end{tabular}

homogeneous, isotropic turbulence (HIT) at a Taylor Reynolds number of 433 in Fig. 1, with the discriminant function, $\Delta_{L}$ as a solid line and the six regions that are delimited throughout this study also labelled. The features of this diagram are well-known and of particular prominence is the Vieillefosse tail (Vieillefosse 1984) that forms a 'ridge' to the joint distribution function on the positive $R_{A}$ side. The degree of mass along the tail, close to the origin and in the opposite, $(\mathrm{Q}>0, \mathrm{R}<0)$, quadrant can be shown to be statistically significant features of HIT both with respect to Gaussian, random tensors (Tsinober 2001) and random tensors constrained to local properties (Keylock 2017).

Table 1 summarizes how the velocity gradient tensors for HIT extracted from the Johns Hopkins database used to populate Fig. 1 are distributed over the six regions of the $\mathrm{Q}_{A}-$ $\mathrm{R}_{A}$ diagram and the four feasible states for enstrophy production and strain production. What is very clear is that the two most frequently occupied regions (regions 2 and 6 ), with opposite signs for $Q_{A}$ and $R_{A}$, are both dominated by positive strain production and enstrophy production. However, the relative magnitudes of these terms differ significantly in these two cases to establish the change in sign for $R_{A}$. The only region where tensors with negative values for both enstophy production and strain production are more likely than tensors with both terms positive is region 1 . The opposite region to this (region 4) has a nearly equal probability of the two strain/enstrophy production states, while in the regions adjacent to, but above, the discriminant function (regions 3 and 5) it is seen that the physically feasible combination with different signs for the two production terms is nearly as likely as the case where both are positive. Hence, these latter two regions exhibit intermediate characteristics compared to regions 2 and 4 , and 1 and 6 , respectively. These properties of the VGT are summarized here because much subsequent analysis highlights that the normal and non-normal properties of the tensor vary greatly between these regions.

An important study with regards to modelling the dynamics of the VGT is due to Cantwell (1992), who derived the restricted Euler equations assuming that viscosity could be ignored and that the pressure Hessian acted in an isotropic fashion:

$$
\begin{aligned}
\frac{d \mathrm{Q}_{A}}{d t} & =-3 \mathrm{R}_{A} \\
\frac{d \mathrm{R}_{A}}{d t} & =\frac{2}{3} \mathrm{Q}_{A}^{2} .
\end{aligned}
$$

There are two possible solutions: Given the timescale, $t_{0}=1 / \sqrt{\left|\mathrm{Q}_{A}^{0}\right|}$, for $\mathrm{Q}_{A}^{0}=0, \mathrm{R}_{A}<0$ the solutions evolve to the fixed point, $\mathrm{Q}_{A}=\mathrm{R}_{A}=0$. For any other initial conditions the 
solution evolves towards the Vieillefosse tail, defined as the bottom right quadrant of Fig. 1 along the line $\Delta_{L}=0$. That the majority of points lie close to the origin, or along the Vieillefosse tail in Fig. 1 highlights the relevance of the restricted Euler approximation to the Navier-Stokes problem. However, it is clearly necessary to go beyond this approach to derive a workable model for the velocity gradient tensor dynamics that includes terms that prevent the mass of the distribution function over-accumulating at the extreme of the Vieillefosse tail (Wilczek \& Meneveau 2014; Johnson \& Meneveau 2016). This implies that, in addition to $\mathrm{Q}_{A}$ and $\mathrm{R}_{A}$, i.e. normal terms defined in terms of the eigenvalues, it is important to study how non-normality contributes to these dynamics, which is the emphasis of this paper. Thus, we define non-normality and develop our decomposition in the next section.

\section{An additive decomposition of the velocity gradient tensor resolving normal and non-normal effects explicitly}

\subsection{Tensor non-normality and the Schur transform}

This study proposes an additive decomposition of the velocity gradient tensor, A, into normal, B, and non-normal, C, components before any subsequent decomposition into rotation or strain tensors. This permits the unpacking of a number of phenomena commonly lumped together, clarifying the behaviour of the tensor.

If $A$ is normal, then

$$
\mathrm{AA}^{*}=\mathrm{A}^{*} \mathrm{~A} .
$$

An eigenvalue decomposition is given by

$$
\mathrm{L} \wedge \mathrm{L}^{-1}=\mathrm{A},
$$

where $L$ contains the eigenvectors and $\Lambda$ is a diagonal matrix of eigenvalues $\left(\Lambda_{1,1}=\lambda_{1}\right)$. The Schur transform (Schur 1909) is given by:

$$
\mathrm{UTU}^{*}=\mathrm{A},
$$

where $\mathrm{U}$ is unitary and $\mathrm{T}=\Lambda+\mathrm{N}$, with $\mathrm{N}$ a strictly upper triangular tensor for the complex Schur transform. That is, because the $\lambda_{i}$ may contain a conjugate pair (where $\Delta_{L}>0$ ), to ensure $\mathrm{N}$ is upper triangular rather than quasi-upper triangular, a complex Schur decomposition is used throughout this study (Golub \& van Loan 2013). For a brief overview of formal properties of the Schur decomposition, see the appendix.

Clearly, because $U$ is unitary, $\mathrm{UU}^{*}=\mathrm{I}$, where $\mathrm{I}$ is the identity matrix, and this implies $\mathrm{UU}^{-1}=\mathrm{I}$ too. The eigenvalue decomposition sacrifices this unitarity of basis for diagonality of $\Lambda$. Hence, informally, deviations of $L$ from a unitary form in an eigenvalue decomposition are reflected in $\mathrm{N}$ in the Schur decomposition. Therefore, the advantage of the Schur decomposition is that it moves non-normal effects out of the eigenvectors (orientations) and into a tensor $\mathrm{N}$ that may be additively coupled to the eigenvalues. From (2.1) and this discussion it follows that $\left\|A^{*}-A^{*} A\right\|$ and $\|N\|$ are both measures of non-normality (Henrici 1962; Eberlein 1965; Lee 1995).

\subsection{Normal and non-normal velocity gradient tensors and the invariants of the velocity} gradient tensor

The normal and non-normal tensors may now be stated:

$$
\begin{aligned}
& \mathrm{B}=\mathrm{U} \wedge \mathrm{U}^{*} \\
& \mathrm{C}=\mathrm{UNU}^{*},
\end{aligned}
$$


and this is the key conceptual innovation in this paper. Thus, there is an additive decomposition, $A=B+C$. The dynamics driven by the eigenvalues are in $B$, which from the restricted Euler formulation (Cantwell 1992) are associated with the isotropic part of the pressure Hessian. Hence, the non-normal effects in $\mathrm{C}$ are associated with asymmetries in the VGT induced by viscosity and deviatoric part of the pressure Hessian. This can be shown using the Poisson equation for the pressure to obtain $2 Q_{A}=\operatorname{tr}(H)$. The involvement of a term on the left hand side that is obtained from the eigenvalues for $A$ means we may define the normal, isotropic part of the pressure Hessian as

$$
\mathrm{H}^{i s o}=\frac{2}{3} \mathrm{Q}_{A} \mathrm{I} \equiv \frac{1}{3} \operatorname{tr}(\mathrm{H}) \mathrm{I}
$$

and this term acts locally. Subtracting $\mathrm{H}^{\text {iso }}$ from $\mathrm{H}$ leaves the deviatoric part $\mathrm{H}^{\text {dev }}$. Ohkitani \& Kishiba (1995) showed using the theory of singular integrals that $\mathbf{H}^{\text {dev }}$ is the nonlocal component of $\mathrm{H}$, expressed as a principal value integral of the pressure field in the neighbourhood of the tensor.

Taking the rotation and strain tensors for B and $\mathrm{C}$, gives

$$
\begin{aligned}
& \mathrm{B}=\mathrm{S}_{B}+\Omega_{B} \\
& \mathrm{C}=\mathrm{S}_{C}+\Omega_{C} .
\end{aligned}
$$

In terms of the Frobenius norms, non-normality is partitioned equally across $\mathrm{S}_{C}$ and $\Omega_{C}$, which means that the constitutive terms for $\mathrm{Q}_{A}(1.11)$ may be written as

$$
\begin{aligned}
& \left\|S_{A}\right\|^{2}=\left\|S_{B}\right\|^{2}+\left\|S_{C}\right\|^{2} \equiv\left\|S_{B}\right\|^{2}+\left\|\Omega_{C}\right\|^{2} \\
& \left\|\Omega_{A}\right\|^{2}=\left\|\Omega_{B}\right\|^{2}+\left\|\Omega_{C}\right\|^{2} \equiv\left\|\Omega_{B}\right\|^{2}+\left\|S_{C}\right\|^{2}
\end{aligned}
$$

The importance of this is seen with respect to eq. (1.11): the second invariant may now be written as

$$
\mathrm{Q}_{A} \equiv \mathrm{Q}_{B}=\frac{1}{2}\left(\left\|\Omega_{B}\right\|^{2}-\left\|\mathrm{S}_{B}\right\|^{2}\right)
$$

where the component terms are smaller by a factor of $\left\|S_{C}\right\|^{2}=\left\|\Omega_{C}\right\|^{2}$.

Recalling that $\mathrm{R}_{A}=-\operatorname{det}(\mathrm{A}) \equiv \prod \lambda_{i}$ and the eigenvalues of $\mathrm{A}$ and $\mathrm{B}$ are identical, then it must also follow that $\mathrm{R}_{A}=\mathrm{R}_{B}$. Thus, the third invariant becomes

$$
\begin{aligned}
\mathrm{R}_{B} & =-\operatorname{det}\left(\mathrm{S}_{B}\right)-\operatorname{tr}\left(\Omega_{B}^{2} \mathrm{~S}_{B}\right) \\
& =\mathrm{R}_{B}^{(S)}-\operatorname{tr}\left(\Omega_{B}^{2} \mathrm{~S}_{B}\right),
\end{aligned}
$$

where the additional terms involving $C$ still need to be determined. These will appear on both sides of the difference in (2.12) so that $\mathrm{R}_{A} \equiv \mathrm{R}_{B}$, but $\mathrm{R}_{A}^{(S)} \neq \mathrm{R}_{B}^{(S)}$ except for where $A$ is normal and $A=B$.

\subsection{Some physical aspects of this decomposition}

From the above identities, $\mathrm{Q}_{A} \equiv \mathrm{Q}_{B}$, and $\mathrm{R}_{A} \equiv \mathrm{R}_{B}$ it follows that the Cantwell (1992) restricted Euler model for the VGT dynamics in $(1.14,1.15)$ only describes the behaviour of the normal part of the VGT, as can be seen from the use of $\mathrm{H}^{i s o}$ in their derivation. Thus, studying the component terms of the second and third invariants when $\|C\| \neq 0$ will introduce non-local effects into consideration. A further important aspect of the approach articulated here is that the discriminant, $\Delta_{L}$ has an explicit role in the analysis of rotation, which is not the case when one studies $\left\|S_{A}\right\|$ and $\left\|\Omega_{A}\right\|$. While the discriminant function defines a sharp threshold between real eigenvalue regions and those with a conjugate pair (and closed streamlines in the Lagrangian frame), non-zero values for $\mathrm{S}_{A}$ and $\Omega_{A}$ may occur throughout the $\mathrm{Q}_{A}-\mathrm{R}_{A}$ space (although, of course, their relative 
magnitudes change). With the Schur approach, because B is an eigenvalue-based tensor, $\Delta_{L}=0$ demarcates a change in one of our rotation tensors. Mathematically, this arises because the eigenvalues for $S_{B}$ and $\Omega_{B}$ are the real and imaginary parts of the eigenvalues for $A$. Hence, where $\Delta_{L}<0$ there is no imaginary part and $\left\|\Omega_{B}\right\|=0$, i.e. there is no rotation in the normal part of the tensor. Therefore, $\mathrm{Q}_{B}=-\frac{1}{2}\left\|\mathrm{~S}_{B}\right\|^{2}$ and all enstrophy comes from $C$. As a consequence of $\left\|\Omega_{B}\right\|=0, \operatorname{tr}\left(\Omega_{B}^{2} S_{B}\right)=0$ and the eigenvalues for $R_{B}$ and $\mathrm{R}_{B}^{(S)}$ are identical. Hence, the third invariant also has a simple expression beneath the discriminant function in this case: $\mathrm{R}_{A} \equiv \mathrm{R}_{B}=\mathrm{R}_{B}^{(S)}$.

Above the discriminant function, while the values for the third invariant of $A$ are no longer equated with those for $\mathrm{R}_{B}^{(S)}$, there is a still a relation between the strain tensor for B and B itself: $\operatorname{sgn}\left(\mathrm{R}_{B}^{(S)}\right)=\operatorname{sgn}\left(\mathrm{R}_{B}\right)$. From the eigenvalue structure, it follows that

$$
\operatorname{tr}\left(\Omega_{B}^{2} S_{B}\right)=\operatorname{Im}\left(\lambda_{c}\right)^{2} \lambda_{r}
$$

where the $r$ and $c$ subscripts indicate the real and conjugate pair eigenvalues for $A$ (and $\mathrm{B}$ ). Thus, $\operatorname{sgn}\left(\operatorname{tr}\left(\Omega_{B}^{2} \mathrm{~S}_{B}\right)\right)=-\operatorname{sgn}\left(\mathrm{R}_{B}^{(S)}\right)$. This means the nature of the normal contributions to strain production and enstrophy production are known from the identified regions of the $\mathrm{Q}_{A}-\mathrm{R}_{A}$ diagram. Observed departures from such relations for $\operatorname{tr}\left(\Omega_{A}^{2} \mathrm{~S}_{A}\right)$ and $-\operatorname{det}\left(S_{A}\right)$, as identified in Table 1 , must be a consequence of the dominant influence of terms involving non-normal effects.

\subsection{Evolution equations for the strain and rotation of $\mathrm{B}$ and $\mathrm{C}$}

It is now helpful to write down the equations for the Lagrangian evolution of strain and rotation for $\mathrm{B}$ and $\mathrm{C}$ :

$$
\begin{aligned}
& \frac{\partial \mathrm{S}_{B}}{\partial t}+\mathrm{S}_{B}^{2}+\Omega_{B}^{2}=-\frac{1}{\rho} \mathrm{H}^{i s o}+\nu \nabla^{2} \mathrm{~S}_{B} \\
& \frac{\partial \mathrm{S}_{C}}{\partial t}+\mathrm{S}_{C}^{2}+\Omega_{C}^{2}+\mathrm{S}_{B} \mathrm{~S}_{C}+\mathrm{S}_{C} \mathrm{~S}_{B}+\Omega_{B} \Omega_{C}+\Omega_{C} \Omega_{B}=-\frac{1}{\rho} \mathrm{H}^{d e v}+\nu \nabla^{2} \mathrm{~S}_{C} \\
& \frac{\partial \Omega_{B}}{\partial t}+\Omega_{B} \mathrm{~S}_{B}+\mathrm{S}_{B} \Omega_{B}=\nu \nabla^{2} \Omega_{B} \\
& \frac{\partial \Omega_{C}}{\partial t}+\Omega_{C} \mathrm{~S}_{C}+\mathrm{S}_{C} \Omega_{C}+\Omega_{B} \mathrm{~S}_{C}+\mathrm{S}_{C} \Omega_{B}+\Omega_{C} \mathrm{~S}_{B}+\mathrm{S}_{B} \Omega_{C}=\nu \nabla^{2} \Omega_{C}
\end{aligned}
$$

For each expression in (2.14) we can multiply the term in the time derivative by itself, take the trace of the expression and divide by -2 . The Cayley-Hamilton theorem, $\operatorname{det}(A)=\frac{1}{3} \operatorname{tr}\left(A^{3}\right)$ can then be used to obtain an expression for the second invariants of each of these terms. Thus, for $S_{B}$ we have:

$$
\frac{\partial \mathrm{Q}_{B}^{(S)}}{\partial t}+\frac{1}{2} \mathrm{R}_{B}+\mathrm{R}_{B}^{(S)}=-\frac{1}{2} \nu \operatorname{tr}\left(\mathrm{S}_{B} \nabla^{2} \mathrm{~S}_{B}\right)
$$

where the zero trace for $S_{B}$ sets $-\frac{1}{\rho} \operatorname{tr}\left(H^{i s o} S_{B}\right)=0$. The equation for $S_{C}$ is

$$
\frac{\partial \mathrm{Q}_{C}^{(S)}}{\partial t}+\mathrm{R}_{C}^{(S)}-\operatorname{tr}\left(\mathrm{S}_{C}^{2} \mathrm{~S}_{B}\right)=-\frac{1}{2}\left[\frac{1}{\rho} \operatorname{tr}\left(\mathrm{H}^{d e v} \mathrm{~S}_{C}\right)+\nu \operatorname{tr}\left(\mathrm{S}_{C} \nabla^{2} \mathrm{~S}_{C}\right)\right]
$$

where $\mathrm{R}_{C}^{(S)} \equiv-\operatorname{det}\left(\mathrm{S}_{C}\right)=\operatorname{tr}\left(\Omega_{C}^{2} \mathrm{~S}_{C}\right)$ has been used to eliminate the latter term. Hence, this highlights that the evolution of non-normal production is a consequence of stretching by both $S_{C}$ and $S_{B}$. 
TABLE 2. The strain production and enstrophy production terms in this study.

Term Equivalent Interpretation

$\mathrm{R}_{B}^{(S)} \quad$ - $\quad$ self-amplification of normal strain (normal strain production)

$\operatorname{tr}\left(\Omega_{B}^{2} S_{B}\right)$ - normal enstrophy production

$\mathrm{R}_{C}^{(S)} \operatorname{tr}\left(\Omega_{C}^{2} \mathrm{~S}_{C}\right) \quad$ self-amplification of non-normality (non-normal production)

$\operatorname{tr}\left(\Omega_{C}^{2} S_{B}\right)-\operatorname{tr}\left(S_{C}^{2} S_{B}\right)$ normal straining of non-normality (interaction production)

The rotation terms may be written as

$$
\frac{\partial \mathrm{Q}_{B}^{(\Omega)}}{\partial t}+\mathrm{R}_{B}+\mathrm{R}_{B}^{(S)}=-\frac{1}{2} \nu \operatorname{tr}\left(\Omega_{B} \nabla^{2} \Omega_{B}\right),
$$

and

$$
\frac{\partial \mathrm{Q}_{C}^{(\Omega)}}{\partial t}-\mathrm{R}_{C}^{(S)}-\operatorname{tr}\left(\Omega_{C}^{2} \mathrm{~S}_{B}\right)=-\frac{1}{2} \nu \operatorname{tr}\left(\Omega_{C} \nabla^{2} \Omega_{C}\right) .
$$

Given that $\mathrm{Q}_{C}^{(S)}=-\mathrm{Q}_{C}^{(\Omega)}$ as can be shown using (1.6), (1.11) and (2.10), it follows that either (2.16) or (2.18) may be removed. Adding these two equations together gives

$$
\frac{1}{\rho} \operatorname{tr}\left(\mathrm{H}^{\operatorname{dev}} \mathrm{S}_{C}\right)=\nu\left[\operatorname{tr}\left(\Omega_{C} \nabla^{2} \Omega_{C}-\operatorname{tr}\left(\mathrm{S}_{C} \nabla^{2} \mathrm{~S}_{C}\right)\right] .\right.
$$

In other words, non-normal straining of the non-local effects in the pressure Hessian equates to the dissipation due to the action of $\mathrm{C}$.

The four terms that emerge in equations (2.15-2.18) are summarized in Table 2. It is now clear that the component terms in the strain production - enstrophy production balance for $\mathrm{R}_{B}$ in (2.12) differ from those for $\mathrm{R}_{A}$ in (1.12) by $\mathrm{R}_{C}^{(S)}+\operatorname{tr}\left(\Omega_{C}^{2} \mathrm{~S}_{B}\right)$. Thus, the strain production and enstrophy production terms in (1.12) are

$$
\begin{aligned}
\mathrm{R}_{A}^{(S)} & \equiv-\operatorname{det}\left(\mathrm{S}_{A}\right)=\mathrm{R}_{B}^{(S)}+\mathrm{R}_{C}^{(S)}+\operatorname{tr}\left(\Omega_{C}^{2} \mathrm{~S}_{B}\right) \\
\operatorname{tr}\left(\Omega_{A}^{2} \mathrm{~S}_{A}\right) & =\operatorname{tr}\left(\Omega_{B}^{2} \mathrm{~S}_{B}\right)+\mathrm{R}_{C}^{(S)}+\operatorname{tr}\left(\Omega_{C}^{2} \mathrm{~S}_{B}\right) .
\end{aligned}
$$

Essentially, the remainder of this paper studies the nature of these terms and their interactions as a function of the region of the $\mathrm{Q}_{A}-\mathrm{R}_{A}$ diagram. However, because this study also looks at some topological and alignment properties of the tensor in the results sections, the Schur framework is first used to rewrite the usual measures for studying these properties of the VGT as detailed below.

\subsection{The second strain eigenvalue and its Lund and Rogers normalization}

One property of HIT that was observed in the early simulations was a strong preference for a positive second eigenvalue of the strain rate tensor (Kerr 1985; Ashurst et al. 1987). This may be inferred from the shape of the $\mathrm{Q}_{A}-\mathrm{R}_{A}$ diagram in Fig. 1 and, in particular, the values in Table 1 that indicate a preference for positive strain production and, thus, two positive strain eigenvalues. Topologically, this means that flow packets are more prone to evolve to disc-like features than rod-like features.

The Lund and Rogers normalization of the second eigenvalue of the strain rate tensor 
is used to study this property of turbulence, and is given by (Lund \& Rogers 1994):

$$
e_{A}^{(L R)}=\frac{3 \sqrt{6} \mathrm{R}_{A}^{(S)}}{\left(-2 \mathrm{Q}_{A}^{(S)}\right)^{\frac{3}{2}}},
$$

which is bounded to $-1 \leqslant e_{A}^{(L R)} \leqslant 1$. Similar measures for the second strain eigenvalue for $\mathrm{B}$ and $\mathrm{C}$ then follow, e.g. $e_{B \mid A}^{(L R)}=3 \sqrt{6} \mathrm{R}_{B}^{(S)} /\left(-2 \mathrm{Q}_{A}^{(S)}\right)^{\frac{3}{2}}$. However, from (2.20), the formulation for $\mathrm{R}_{A}^{(S)}$ also contains an interaction term: $\mathrm{R}_{A}^{(S)}=\mathrm{R}_{B}^{(S)}+\mathrm{R}_{C}^{(S)}+\operatorname{tr}\left(\Omega_{C}^{2} \mathrm{~S}_{B}\right)$. This results in the interaction metric

$$
e_{C, B \mid A}^{(L R)}=\frac{3 \sqrt{6} \operatorname{tr}\left(\Omega_{C}^{2} S_{B}\right)}{\left(-2 Q_{A}^{(S)}\right)^{\frac{3}{2}}},
$$

leading to

$$
e_{A}^{(L R)}=e_{B \mid A}^{(L R)}+e_{C \mid A}^{(L R)}+e_{C, B \mid A}^{(L R)} .
$$

Section 2.3 describes how the strain and enstrophy for $B$ are constrained by the eigenvalues and, therefore their signs are given by the regions of the $\mathrm{Q}_{A}-\mathrm{R}_{A}$ diagram. Thus, it is known a priori that $0<e_{B \mid A}^{(L R)} \leqslant 1$ on the $\mathrm{R}_{A}>0$ side of the diagram and $-1 \leqslant e_{B \mid A}^{(L R)}<0$ on the negative side. This additive decomposition of $e_{A}^{(L R)}$ is used in Section 5 to determine how each term contributes to the rod or disc topology.

\subsection{Alignment properties of the vorticity vector and the strain eigenvectors}

An important and surprising early result in the study of the velocity gradient tensor was the preferred alignment between the vorticity vector, $\boldsymbol{\omega}_{A}$ and the eigenvector for the intermediate eigenvalue of the strain rate tensor (Kerr 1985; Ashurst et al. 1987; Jimenez 1992). The approach taken in this study gives vorticity vectors for A, B, and C, as well as three strain eigenvectors for each of the three tensors. This means that the Schur decomposition approach has the potential to clarify how these alignments originate. Thus, with $e_{i}^{A}$ indicating an eigenvalue of the strain rate tensor for $\mathrm{A}$, ordered from most positive to most negative, and $\mathbf{e}_{i}^{A}$ its corresponding eigenvector, the notation adopted here for a vorticity-strain alignment is given by

$$
\theta_{i}^{A, C}=\cos \left(\boldsymbol{\omega}_{A}, \mathbf{e}_{i}^{C}\right),
$$

where this example is the angle between the vorticity vector for A and the $i$ th eigenvector for C. Furthermore, there are the mutual strain alignments, e.g.:

$$
\phi_{i, j}^{A, B}=\cos \left(\mathbf{e}_{i}^{A}, \mathbf{e}_{j}^{B}\right),
$$

and the mutual vorticity vector alignments, such as

$$
\xi^{A, B}=\cos \left(\boldsymbol{\omega}^{A}, \boldsymbol{\omega}^{B}\right) .
$$

\section{The Numerical Simulation}

This study makes use of velocity gradient tensors extracted from the Johns Hopkins Turbulence Database numerical simulation of forced isotropic turbulence at a Taylor Reynolds number of 433 (Li et al. 2008) as described by Wan et al. (2016). The direct numerical simulation is undertaken on a $1024^{3}$ grid using a pseudo-spectral method. The 
TABle 3. Properties of the HIT simulation in the Johns Hopkins database (Li et al. 2008).

\section{Property \\ Grid}

Domain

Viscosity, $\nu$

Mean dissipation rate, $\epsilon$

Taylor micro-scale, $\lambda$

Taylor Reynolds number, $R e_{\lambda}$

Kolmogorov length, $\eta$

\section{Value}

$1024^{3}$ periodic box

$[0,2 \pi]^{3}$

$1.85 \times 10^{-4}$

0.0928

0.118

433

$2.87 \times 10^{-3}$
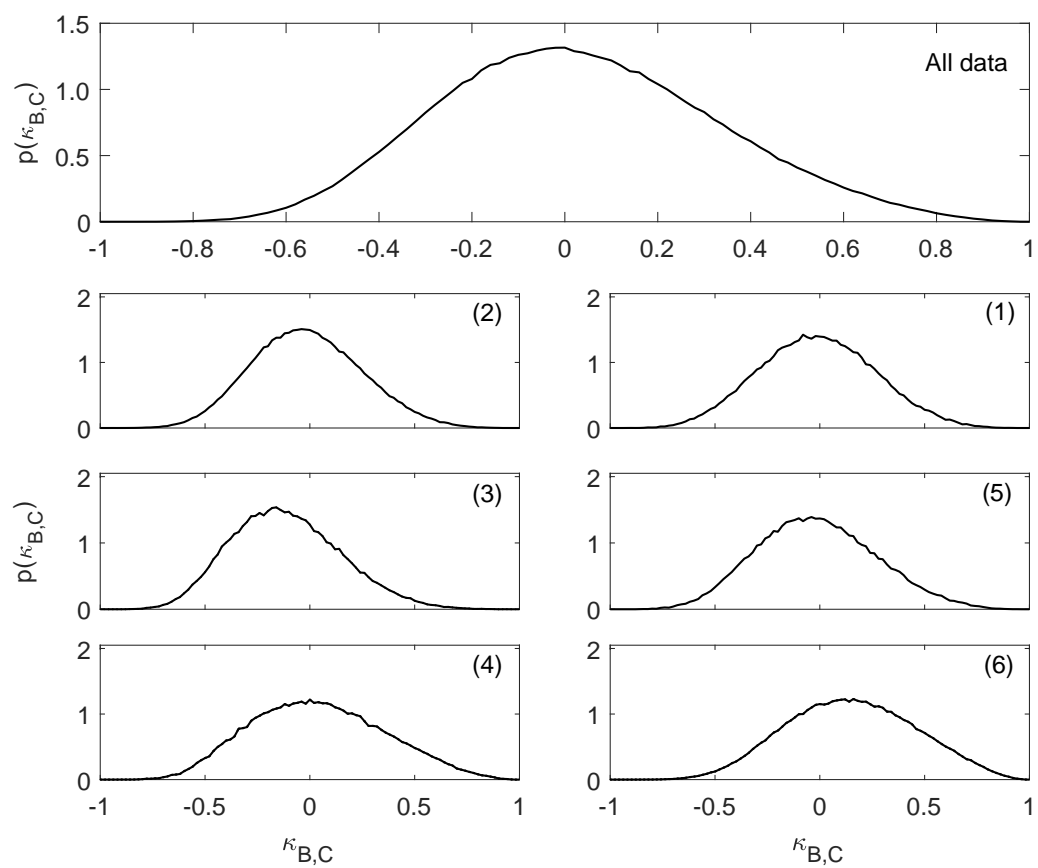

Figure 2. The probability curves for $\kappa_{B, C}$, the normalized difference in the Frobenius norms for $B$ and $C$, shown for all the data and as a function of the six regions of the $Q_{A}-R_{A}$ diagram in the various panels.

energy is injected to maintain the total energy in the Fourier modes, and also retaining a wave number magnitude less than or equal to 2 in each mode. The basic properties of the simulation are summarized in Table 3 and a number of other studies have made use of this resource for studying turbulence physics (Wan et al. 2010; Lawson \& Dawson 2015) or for the testing of data post-processing algorithms (Higham et al. 2016).

\section{Results: The role of non-normality}

\subsection{The importance of non-normal effects}

Given $A=B+C$, the first thing to establish is the relative importance of the normal and non-normal tensors; clearly if $C$ is small then it is legitimate to approximate the behaviour of $\mathrm{A}$ with eigenvalue-based formulations. A standardized difference can be 


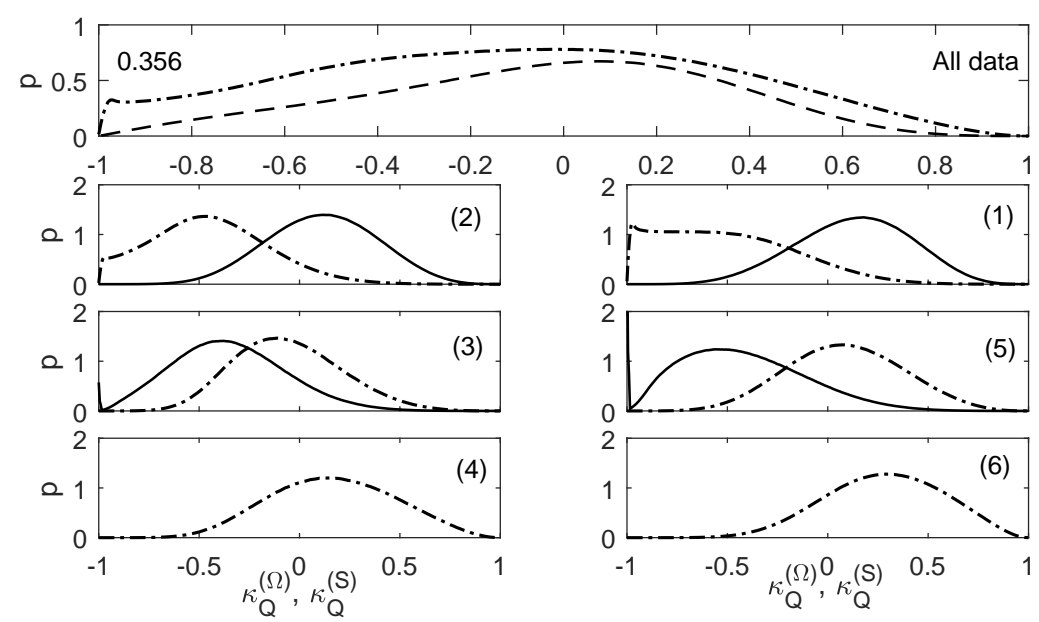

Figure 3. The distributions for $\kappa_{Q}^{(\Omega)}$ (solid lines) and $\kappa_{Q}^{(S)}$ (dot-dashed lines), the normalized differences in the Frobenius norms for $\Omega_{B}$ and $S_{B}$, respectively, compared to the non-normal term, $\mathrm{S}_{C}$. Results are shown for all the data and as a function of the six regions of the $\mathrm{Q}_{A}-\mathrm{R}_{A}$ diagram. The dashed line in the top panel gives the results for $\kappa_{Q}^{(\Omega)}$ with the data at $\kappa_{Q}^{(\Omega)}=-1$ from regions 4 and 6 excluded. Altogether, $35.6 \%$ of values lie at this $\kappa_{Q}^{(\Omega)}=-1$ limit as stated in this panel.

used to evaluate this

$$
\kappa_{B, C}=\frac{\|\mathrm{B}\|-\|\mathrm{C}\|}{\|\mathrm{B}\|+\|\mathrm{C}\|} .
$$

This term is shown in Fig. 2 and it is clear from the upper panel that the overall mode for the distribution is slightly negative, with a median close to $\kappa_{B, C}=0$. Hence, the non-normality is as important to the tensor as the part explained by the eigenvalues. Thus, in HIT, asymmetrical forcings on the tensor as a consequence of non-local effects or viscous effects are an important part of the flow dynamics. When the results in Fig. 2 are partitioned by the six regions of the $\mathrm{Q}_{A}-\mathrm{R}_{A}$ diagram, it is clear that for each pair of diagrams in a given $\mathrm{Q}_{A}$ state, it is the left-hand case, with $\mathrm{R}_{A}<0$, where the contribution from $\|\mathrm{C}\|$ is greater. Region 3 is where $\kappa_{B, C}$ is most strongly negative and region 6 is where $\kappa_{B, C}$ is most positive. Thus, an eigenvalue-based description of the flow is particularly effective near the Vieillefosse tail, which is consistent with this being an attractor for the dynamics of the restricted Euler (eigenvalue-based) set of equations for the dynamics of the VGT (Cantwell 1992).

\subsection{The second invariant and non-normality}

From (2.11), the components of the second invariant with respect to non-normality are

$$
\begin{gathered}
\kappa_{Q}^{(\Omega)}=\frac{\left\|\Omega_{B}\right\|-\left\|S_{C}\right\|}{\left\|\Omega_{B}\right\|+\left\|S_{C}\right\|} \\
\kappa_{Q}^{(S)}=\frac{\left\|S_{B}\right\|-\left\|S_{C}\right\|}{\left\|S_{B}\right\|+\left\|S_{C}\right\|} .
\end{gathered}
$$

Beneath the discriminant function, $\kappa_{Q}^{(\Omega)}=-1$ by definition and such results are not shown in the panels for regions (4) and (6) in Fig. 3. While the expected increase in the mean values for $\kappa_{Q}^{(\Omega)}$ and decrease for $\kappa_{Q}^{(S)}$ with increasing $\mathrm{Q}_{A}$ is very distinct, the 


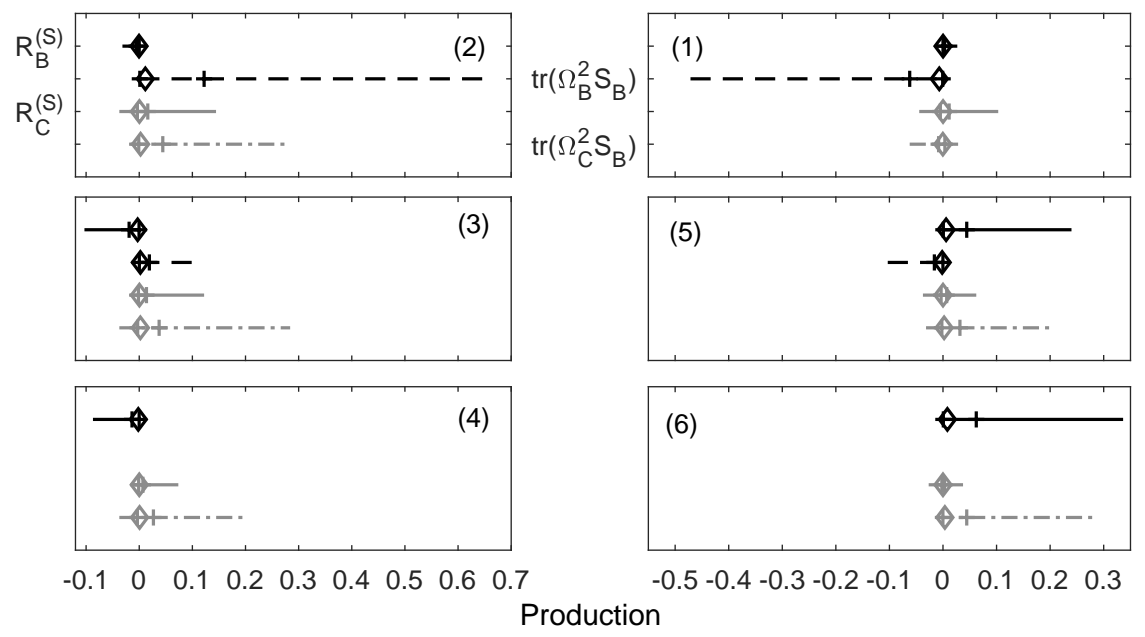

FiguRE 4. The range of production values from the first percentile to the 99th percentile is shown for each of the four production terms conditioned on the region of the $Q_{A}-R_{A}$ diagram (indicated in brackets in each panel). The median value is indicated by a diamond and, where visible the $10 \%$ and $90 \%$ values are shown by a vertical tick. Values are non-dimensionalized by the Kolmogorov time cubed. The black lines are the normal terms and the grey are the non-normal and interaction terms; which specific term is examined is indicated in the top two panels.

differences as a function of the sign of $\mathrm{R}_{A}$ are also clearer than in Fig. 2. For example, while a difference between regions 4 and 6 in their values for $\kappa_{Q}^{(S)}$ is anticipated from the discussion of Fig. 2, their respective modes of 0.16 and 0.28 indicate this very clearly. In regions 3 and 5 , which is where $\Delta_{L}>0$ and $\mathrm{Q}_{A}<0$, there is a positive mode for region 5 at $\kappa_{Q}^{(S)}=0.07$ and a mode of $\kappa_{Q}^{(S)}=-0.12$ for region 3 . Where $\mathrm{Q}_{A}>0$, the mode in region 1 for $\kappa_{Q}^{(\Omega)}=0.18$ is more positive than for region $2\left(\kappa_{Q}^{(\Omega)}=0.12\right)$, and there is also a stronger tendency for $\kappa_{Q}^{(S)} \rightarrow-1$. The importance of non-normal effects in the vortical regions (1 and 2) suggests that a number of properties of vortical structures, such as their topology and alignments, are poorly described by the eigenvalues. Hence, this motivates the additional sections below concerning these aspects of turbulence.

\subsection{The third invariant and the four production terms}

The components of the third invariant are listed and interpreted in Table 2 and the probability distributions for the four production terms are synthesized in Fig. 4, conditioned on the region of the $\mathrm{Q}_{A}-\mathrm{R}_{A}$ diagram, with values non-dimensionalized by the cube of the Kolmogorov time. As explained in Section 2.3, $\operatorname{sgn}\left(\mathrm{R}_{B}^{(S)}\right)=\operatorname{sgn}\left(\mathrm{R}_{A}\right)$ and $\operatorname{sgn}\left(\operatorname{tr}\left(\Omega_{B}^{2} S_{B}\right)\right)=-\operatorname{sgn}\left(R_{A}\right)$, while $\operatorname{tr}\left(\Omega_{B}^{2} S_{B}\right)=0$ beneath the discriminant function (regions 4 and 6). Thus, the basic properties of these terms (the black lines in Fig. 4 are known directly from the Schur formalism.

It is clear from Fig. 4 that both $\mathrm{Q}_{A}>0$ and $\Delta_{L}>0$ are relevant to classifying the flow behaviour, justifying the separation of the $\mathrm{Q}_{A}-\mathrm{R}_{A}$ diagram into six regions. First, $\operatorname{tr}\left(\Omega_{B}^{2} S_{B}\right)$ does not occur for $\Delta_{L}<0$ as just described and, second, all other terms exhibit a clearer difference between positive and negative $Q_{A}$ states than positive and negative $\Delta_{L}$ states. For example, in regions 5 and 6 , the positive tail of the distributions for both $\mathrm{R}_{B}^{(S)}$ and $\operatorname{tr}\left(\Omega_{C}^{2} \mathrm{~S}_{B}\right)$ is clear. However, in region 1 the latter changes sign and the former 
collapses dramatically. In addition, $\mathrm{R}_{C}^{(S)}$ becomes more positively skewed and $\operatorname{tr}\left(\Omega_{B}^{2} \mathrm{~S}_{B}\right)$ more negatively skewed. That there is little difference between regions 5 and 6 for three of the terms, means that the emergence of negative contributions from $\operatorname{tr}\left(\Omega_{B}^{2} S_{B}\right)$ in region 5 is the sole major difference to the dynamics.

In regions 3 and 4, the non-normal and interaction production terms are broadly similar to regions 5 and 6 but the normal terms are opposite in sign and the tail to the normal strain production term is much shorter. Regions 1 and 2 are qualitatively different both to each other and to the other regions. Clearly, normal enstrophy production dominates in these two regions and is necessarily opposite in sign between them. In addition, nonnormal production, $\mathrm{R}_{C}^{(S)}$ is more positively skewed in these two regions than elsewhere. However, while the interaction term is positively skewed in other regions, in region 1 it exhibits a weak negative skew. As a consequence of the sign for $\operatorname{tr}\left(\Omega_{B}^{2} S_{B}\right)$, positive production in region 1 comes from the non-normal term. Hence, positive values for $\mathrm{R}_{A}$, in region 1 are driven by positive non-normal production and negative normal enstrophy production rather than positive normal production, as is the case in regions 5 and 6 .

A typical Lagrangian trajectory is from region 2 to region 1 , where there is a change from two negative eigenvalues for $\mathrm{R}_{B}^{(S)}$ (rods) to one (discs). However, the small magnitude for $\mathrm{R}_{B}^{(S)}$ means that the non-normal and interaction terms will be important for establishing the values for $\mathrm{R}_{A}^{(S)}$. This is explored in section 5 .

\subsection{Joint behaviour of the four production terms}

Further information on the structure of the production terms can be gleaned from their joint behaviour. Hence, in this section normal strain production is compared to the combined influence of the non-normal and interaction terms (Fig. 5), normal enstrophy production is compared to the combined influence of the non-normal and interaction terms (Fig. 6), and then the joint behaviour of the latter two terms is studied (Fig. 7). Again, each term is non-dimensionalized by the cube of the Kolmogorov time. Each panel in these three figures displays the results for each region of the $\mathrm{Q}_{A}-\mathrm{R}_{A}$ diagram as a difference from the overall joint PDF for all regions combined, with lighter (darker) contours highlighting an excess (deficit) to the distribution function for this region.

There is a basic pattern to these joint distributions anticipated from the signs for $\mathrm{R}_{B}^{(S)}$ and $\operatorname{tr}\left(\Omega_{B}^{2} S_{B}\right)$ on each half of the $Q_{A}-R_{A}$ diagram, with positive strain production and negative enstrophy production occurring on the right hand side and the opposite case seen on the left. However, the sign of $\mathrm{Q}_{A}$ is important for the strength to which this tendency is expressed. The anticipated pattern is seen for strain dominant regions where $\mathrm{Q}_{A}<0$ in Fig. 5 and for enstrophy dominated regions where $\mathrm{Q}_{A}>0$ in Fig. 6. For strain dominated regions in Fig. 6, the combined effect of the non-normal and interaction production terms is essentially positive. For enstrophy dominated regions in Fig. 5, region 2 exhibits an excess for either sign of the combined term, while for region 1 the excess is clearly for negative values of the combined term. It is clear from the results in Fig. 5 for regions 5 and 6 why strain production is highest around the Vieillefosse tail: a strong local excess for positive normal strain production is reinforced by positive contributions from the non-normal and interaction production terms.

Disaggregating the behaviour of this term, Fig. 7 shows that it is the non-normal term that dominates in both regions 1 and 2, and that this can take either sign. Region 6 is very much limited to positive excess contributions from the interaction term, and it is true that it is this term that results in the positive bias for contributions from region 5, although larger magnitude values arise for the non-normal term, reflecting the status of region 5 as intermediary between regions 1 and 6 . Region 3 is the case where 


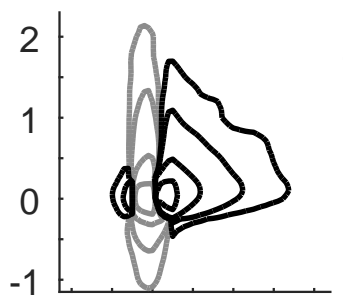

(2)

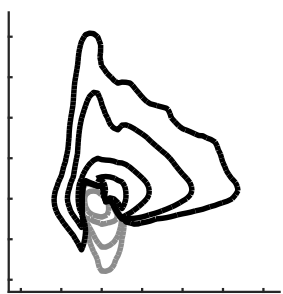

(1)

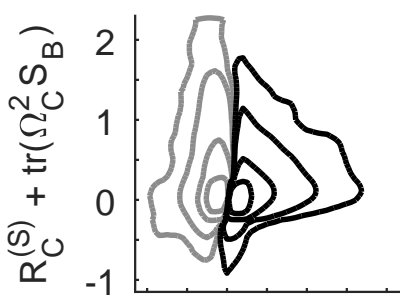

(3)

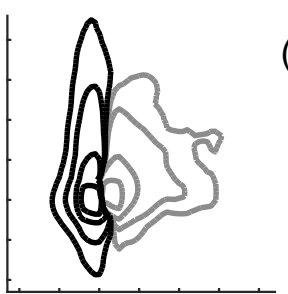

(5)
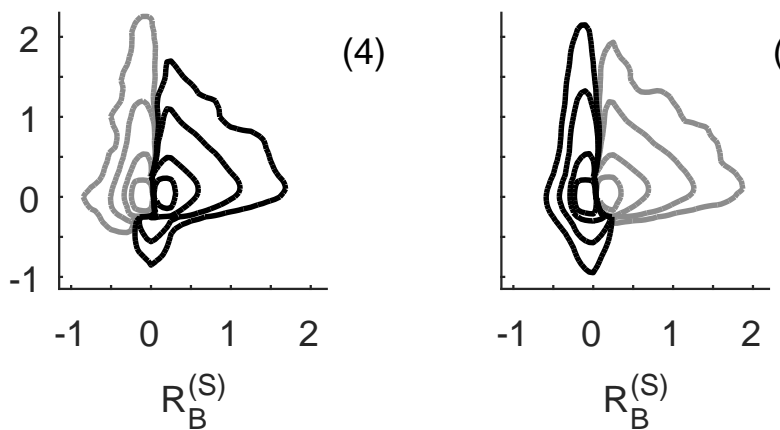

(6)

FIGURE 5. Joint probability distributions for $\mathrm{R}_{B}^{(S)}$ and the combined non-normal and interaction terms conditioned on the region of the $Q_{A}-R_{A}$ diagram, and written as the difference between the distribution in this region and the overall distributions function. Positive values indicate an excess for this region and have the lighter probability contours. Contours are approximately logarithmically distributed: $\pm 2 \times 10^{-3}, \pm 1 \times 10^{-2}, \pm 1 \times 10^{-1}, \pm 1 \times 10^{0}$.

a largely positive response is a consequence of a jointly positive contribution from both terms - this is the region where reducing the effect to one or other of the marginal distributions shown in Fig. 7 is inadequate. Positive contributions from region 4 are a preferential consequence of positive values for $\mathrm{R}_{C}^{(S)}$, irrespective of the sign for $\operatorname{tr}\left(\Omega_{C}^{2} \mathrm{~S}_{B}\right)$. Thus, future models for the VGT dynamics in the spirit of recent approaches (Wilczek \& Meneveau 2014; Johnson \& Meneveau 2016) but including information from this study, may wish to consider stochastic, small-scale forcings from the non-normal contributions in accord with the marginal and joint distributions presented here.

\section{Results: The second eigenvalue of the strain rate tensor for $A$}

The well-known tendency for HIT to form disc-like structures is shown in the upper panel of Fig. 8 using the Lund and Rogers normalization, $e_{A}^{(L R)}$ given in (2.22). Subdividing the results by region of the $\mathrm{Q}_{A}-\mathrm{R}_{A}$ diagram shows that this tendency is driven by regions 2,5 and 6 . However, given that positive strain production, $-\operatorname{det}\left(\mathrm{S}_{A}\right)>0$ means there are two positive strain eigenvalues, inducing disc-like structures in an incompressible 

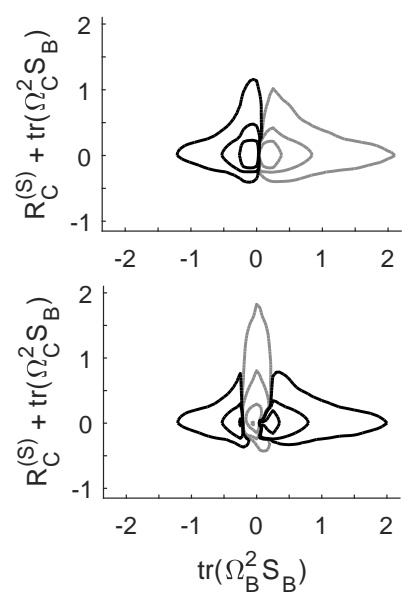

(2)

(3)
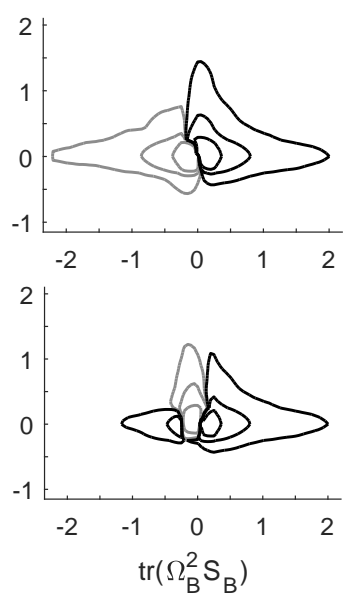

(1)

FiguRE 6. Joint probability distributions for $\operatorname{tr}\left(\Omega_{B}^{2} S_{B}\right)$ and the combined non-normal and interaction terms conditioned on the region of the $Q_{A}-R_{A}$ diagram, and written as the difference between the distribution in this region and the overall distributions function. Positive values indicate an excess for this region and have the lighter probability contours. Contours are logarithmically distributed: $\pm 1 \times 10^{-2}, \pm 1 \times 10^{-1}, \pm 1 \times 10^{0}$.

flow, in regions 1, 5 and 6 , the unusual extent of non-normal production in region 1 , already highlighted, must explain this weak contribution to this tendency from region 1. When looking at $e_{A}^{(L R)}$ conditioned on the sign of $\kappa_{Q}^{(S)}$ (the grey lines in Fig. 8), where $\kappa_{Q}^{(S)}>0$ (solid grey lines) the anticipated behaviour of a tendency for $e_{A}^{(L R)} \rightarrow+1$ in region 1 and $e_{A}^{(L R)} \rightarrow-1$ in region 2 is seen. However, the grey dashed lines, representing $\kappa_{Q}^{(S)}<0$ states arise much more frequently ( $\sim 90 \%$ of cases $)$, meaning they drive the overall pattern in Fig. 8. It is these data that exhibit the strong $e_{A}^{(L R)} \rightarrow+1$ tendency in region 2, and a flat distribution function in region 1 .

Consequently, the reason for the observed behaviour is that the non-normal contribution to the strain tensor is significant and produces a very different behaviour to that driven by the eigenvalues. Given that it is in regions 1 and 2 where enstrophy exceeds strain and, according to the Q-criterion (Hunt et al. 1988; Dubief \& Delcayre 2000), is where there is a coherence to vortical motion, non-normality is crucial for the evolution of coherent, vortical disc-like structures. As contributions from the non-normality tensor, C, do not feature in the restricted Euler formulation of the VGT dynamics, anistropic contributions from the pressure Hessian and viscous effects must be extremely important for this aspect of the topology. From Table 1, region 2 is frequented more than twice as often as region 1 (26.5\% to $11.1 \%$ ), which also helps explain the strong tendency for $e_{A}^{(L R} \rightarrow 1$ seen in the top-most panel.

Figure 3 shows that $\kappa_{Q}^{(S)}>0$ occurs for $39.4 \%, 68.6 \%, 60.6 \%$ and $82.2 \%$ of occurrences in regions 3 to 6 , respectively (i.e. the normal contributions to strain are larger in regions 4 and 6). In Fig. 8 it is region 5 where $\kappa_{Q}^{(S)}>0$ has the strongest tendency to produce values at $e_{A}^{(L R)}=1$, while everywhere but region 1 , one finds that $\kappa_{Q}^{(S)}<0$ preferentially leads to positive values for $e_{A}^{(L R)}$. The complex case is region 4 , where the normal contribution to the strain is strongly dominant, but has a mode at $e_{A}^{(L R)}=-0.08$, indicating that here the preferred state is close to isotropy (the "blob" in the terminology of Kuo \& Corrsin 


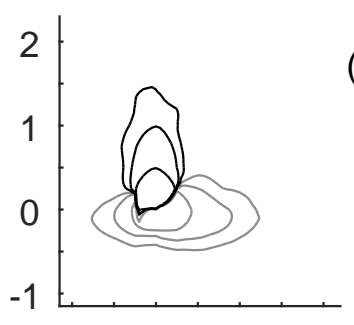

(2)
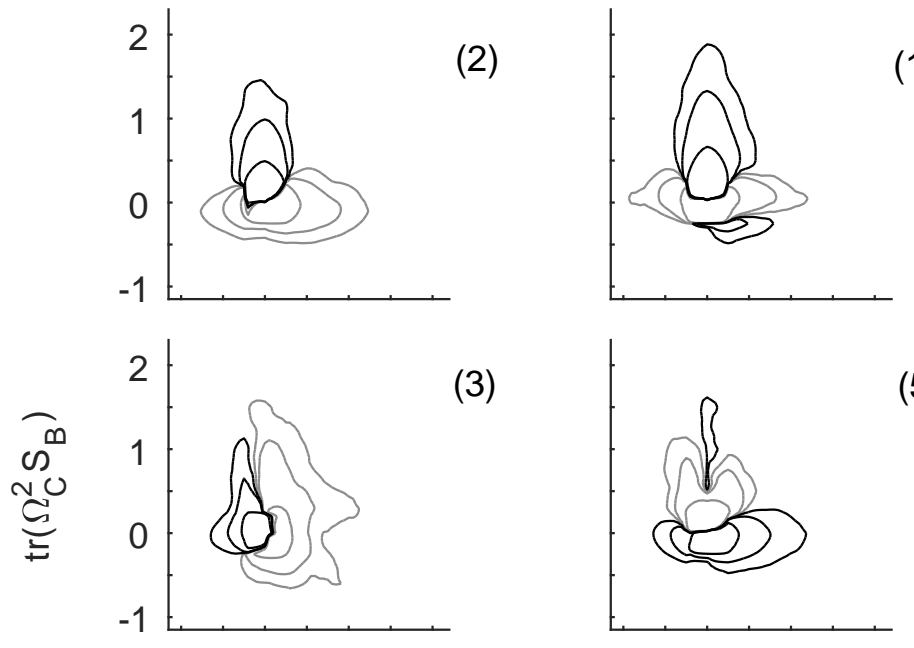

(3)

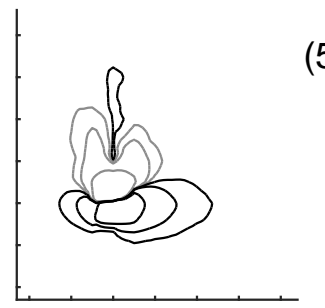

(5)
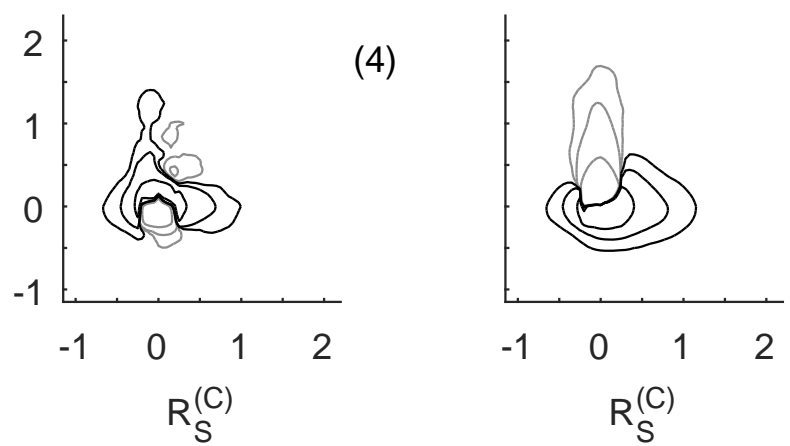

(6)

FIGURE 7. Joint probability distributions for $\mathrm{R}_{C}^{(S)}$ and $\operatorname{tr}\left(\Omega_{C}^{2} \mathrm{~S}_{B}\right)$ conditioned on the region of the $Q_{A}-R_{A}$ diagram, and written as the difference between the distribution in this region and the overall distributions function. Positive values indicate an excess for this region and have the lighter probability contours. Contours are approximately logarithmically distributed: $\pm 2.5 \times 10^{-3}, \pm 1 \times 10^{-2}, \pm 1 \times 10^{-1}$.

(1972)); any tendency to form disc-like structures is a consequence of the non-normal dominant cases in this region.

5.1. The disaggregation of the Lund and Rogers normalization of the strain rate tensor It was established in Section 2.5 that $e_{A}^{(L R)}=e_{B \mid A}^{(L R)}+e_{C \mid A}^{(L R)}+e_{C, B \mid A}^{(L R)}$ and it is the component terms on the right-hand side that we investigate in this sub-section. However,

$$
e_{|A|}^{(L R)}=\left|\frac{e_{A}^{(L R)}}{\left|e_{B \mid A}^{(L R)}\right|+\left|e_{C \mid A}^{(L R)}\right|+\left|e_{C, B \mid A}^{(L R)}\right|}\right|,
$$

may be used to gain an insight into how common it is for some of these component terms to have the opposite sign to $e_{|A|}^{(L R)}$ and the relative magnitude of such effects as a function of $\mathrm{Q}_{A}$ and $\mathrm{R}_{A}$. Given the tendency in HIT for $e_{A}^{(L R)} \rightarrow 1$ and that, as explained in section $2.5, \operatorname{sgn}\left(e_{B \mid A}^{(L R)}\right)=\operatorname{sgn}\left(\mathrm{R}_{A}\right)$, it is expected that $e_{|A|}^{(L R)} \neq 1$ more often in regions 2 , 3, and 4 , where $\mathrm{R}_{A}<0$. Figure 9 shows this is the case, particularly where $\Delta_{L}>0$ in regions 2 


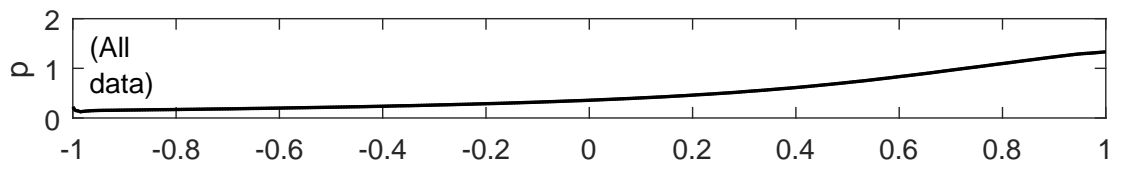

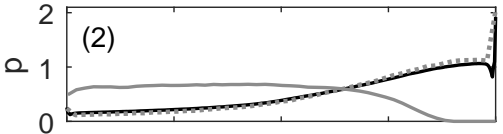
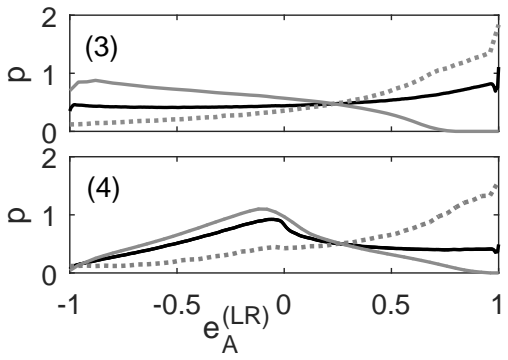
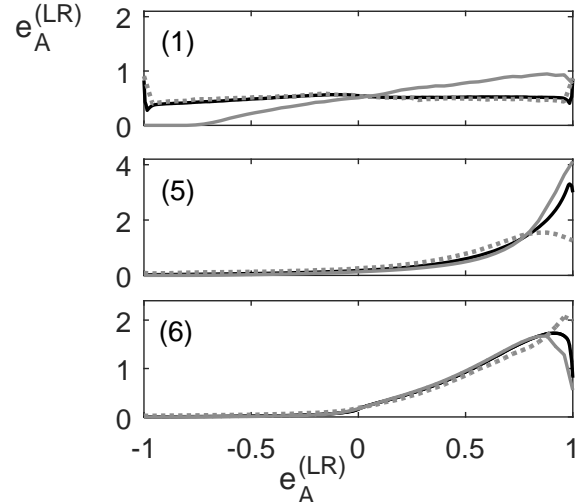

Figure 8. Probability distributions for $e_{A}^{(L R)}$ for all the data (top panel) and then by each region of the $\mathrm{Q}_{A}-\mathrm{R}_{A}$ diagram (black lines). Results are also shown conditioned on the sign of $\kappa_{Q}^{(S)}$, with $\kappa_{Q}^{(S)}>0$ as a grey solid line and $\kappa_{Q}^{(S)}<0$ as a grey dashed line.
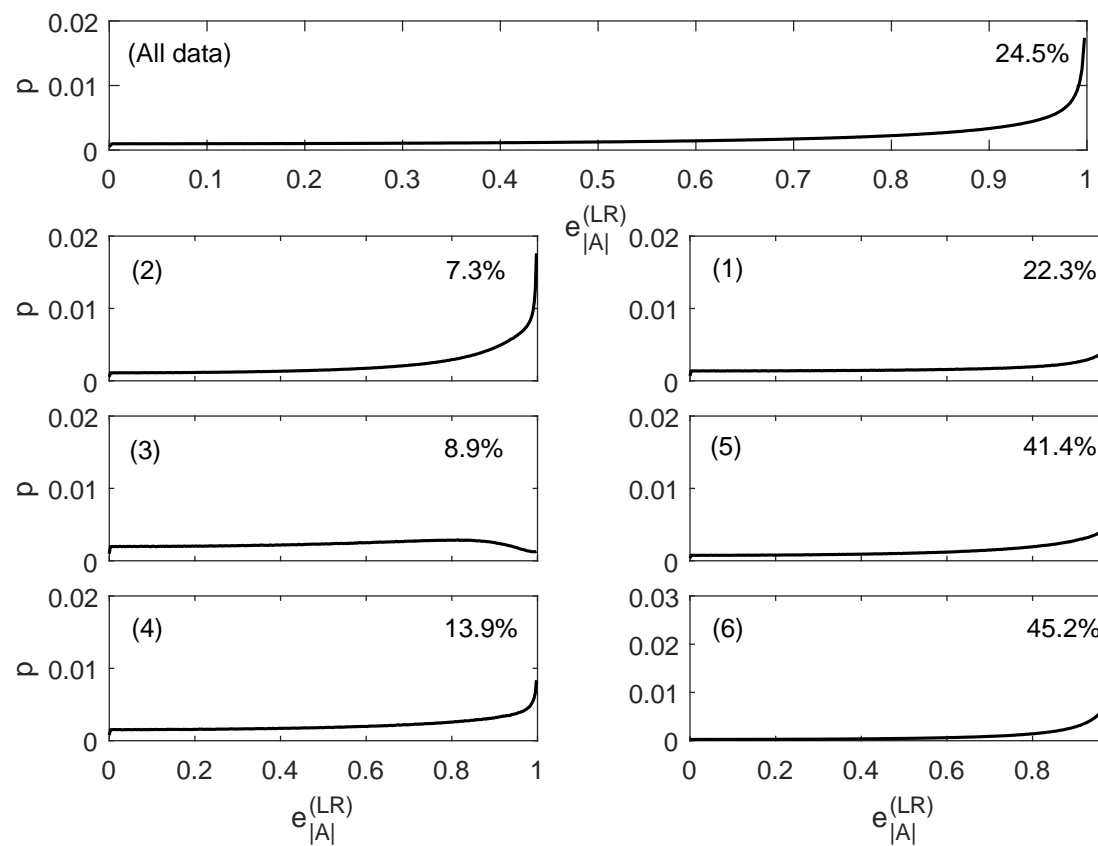

$\mathrm{e}_{|\mathrm{A}|}^{(\mathrm{LR})} 0$

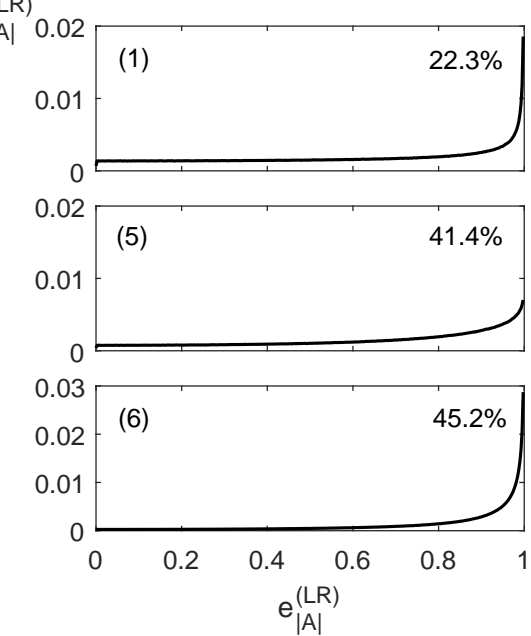

Figure 9. Distribution functions for $e_{|A|}^{(L R)}$ are shown for all the data and by region of the $\mathrm{Q}_{A}-\mathrm{R}_{A}$ diagram. Values are shown with a continuous line for $0 \leqslant e_{|A|}^{(L R)}<1$. The stated value in each panel is the percentage of values for which $e_{|A|}^{(L R)}=1$, i.e. this is where none of the three terms contributing to the value for $e_{A}^{(L R)}$ have a sign different to that for $e_{A}^{(L R)}$. 

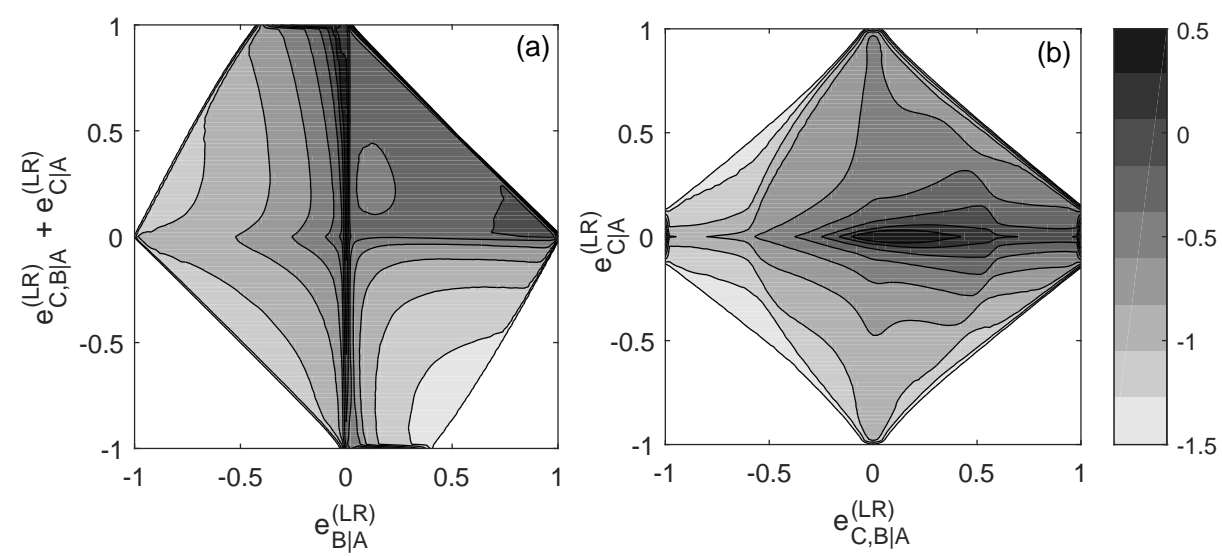

FIgURE 10. Joint distribution functions for $e_{B \mid A}^{(L R)}$ and $e_{C \mid A}^{(L R)}+e_{C, B \mid A}^{(L R)}$ (a) as well as $e_{C \mid A}^{(L R)}$ and $e_{C, B \mid A}^{(L R)}$ (b). Contours are on a $\log _{10}$ scale.

and 3 (and to a certain extent also in region 1 where $\mathrm{R}_{A}>0$ but $\mathrm{Q}_{A}>0$ ). Indeed, with the exception of region 1, the overall result in the upper panel that about $25 \%$ of tensors in HIT have $e_{|A|}^{(L R)}=1$ is not representative of the behaviour of the individual regions.

It follows from these results that the joint distribution function for the normal term, $e_{B \mid A}^{(L R)}$, and the combined non-normal and interaction terms, $e_{C \mid A}^{(L R)}+e_{C, B \mid A}^{(L R)}$, should exhibit the strongest probability gradient near the frontier $e_{B \mid A}^{(L R)}+e_{C \mid A}^{(L R)}+e_{C, B \mid A}^{(L R)}=1$ and where both $e_{B \mid A}^{(L R)}$ and $\left(e_{C \mid A}^{(L R)}+e_{C, B \mid A}^{(L R)}\right)$ are positive. Positive values for $e_{B \mid A}^{(L R)}$ mean the tensor is in region 1, 5 or 6 . Such a frontier is clear in Fig. 10a.

In this same panel, where $e_{B \mid A}^{(L R)}<0$, the combined behaviour of the non-normal and interaction terms behaves with some degree of symmetry about $e_{C \mid A}^{(L R)}+e_{C, B \mid A}^{(L R)}=0$, although with a bias towards positive values as might be expected from the global result of a tendency for $e_{A}^{(L R)} \rightarrow 1$. Therefore, positive values for $e_{A}^{(L R)}$ may readily arise even when $e_{B \mid A}^{(L R)}<0$ as a consequence of the action of the non-normal and interaction terms as shown in a less direct fashion from the conditioning on $\kappa_{Q}^{(S)}$ in Fig. 8.

For positive $e_{B \mid A}^{(L R)}$ negative values for the $e_{C \mid A}^{(L R)}+e_{C, B \mid A}^{(L R)}$ term are highly improbable, except at the limit, $e_{C \mid A}^{(L R)}+e_{C, B \mid A}^{(L R)}=-1$, for weakly positive values for $e_{B \mid A}^{(L R)}$. From an inspection of Fig. 10b, the $e_{C \mid A}^{(L R)}+e_{C, B \mid A}^{(L R)}=-1$ state arises preferentially where $e_{C \mid A}^{(L R)}=0, e_{C, B \mid A}^{(L R)}=-1$, showing the importance of the interaction term for generating rod-like structures. That there is a good degree of symmetry to Fig. $10 \mathrm{~b}$ about $e_{C \mid A}^{(L R)}=0$ indicates that the sign of $e_{C \mid A}^{(L R)}$ is not predicted by $e_{C, B \mid A}^{(L R)}$. That the centre-of-mass of the distribution resides to the right side of Fig. 10b shows that the interaction term is driving the positive bias in values for $e_{A}^{(L R)}$ overall. 


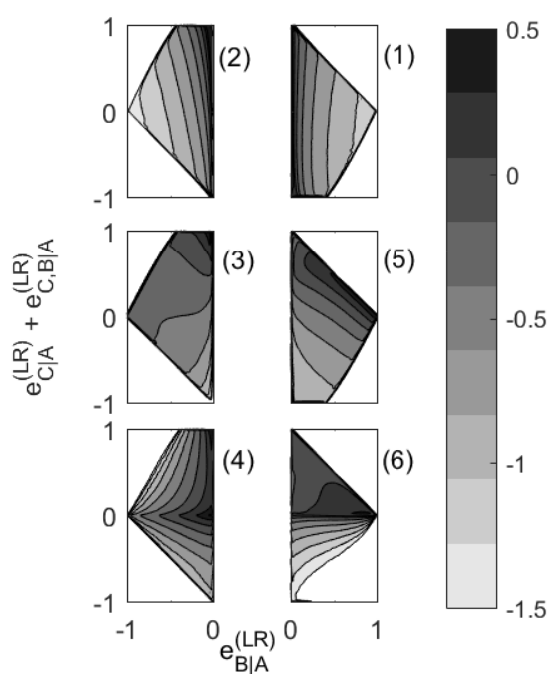

FIGURE 11. Joint distribution functions for $e_{B \mid A}^{(L R)}$ and $e_{C \mid A}^{(L R)}+e_{C, B \mid A}^{(L R)}$ using the same logarithmic scaling for the contours as adopted in Fig. 10, with results sub-divided by the six regions of the $Q_{A}-R_{A}$ diagram defined in Table 1 .

\subsection{Results conditioned on $Q_{A}-R_{A}$ states}

Having presented the general pattern for how the normal, non-normal and interaction terms are coupled in Fig. 10a, results conditioned on the different regions of $\mathrm{Q}_{A}-\mathrm{R}_{A}$ space are shown in Fig. 11. Thus, the strong probability frontier in Fig. 10a and the secondary mode near $e_{B \mid A}^{(L R)}=1$ arise due to the behaviour in regions 5 and 6 , respectively. In contrast $e_{B \mid A}^{(L R)}=-1$ is more common in region 3 than region 4 , and greater relative importance of the non-normal and interaction terms occurs in region 4 compared to region 6 , as $e_{B \mid A}^{(L R)} \rightarrow 0$ in region 4 . The $\mathrm{Q}_{A}-\mathrm{R}_{A}$ diagram exhibits a concentration of values in regions 2 and 6 as shown in Table 1 . Figure 11 shows that the tendency to disc-like structures in region 6 is driven firstly by $e_{B \mid A}^{(L R)}$ and then by the combined interaction of all terms that defines the strong frontier. In contrast, while region 2 contributes effectively to this tendency to form disc-like structures, Fig. 11 shows that this result is driven by $e_{C, B \mid A}^{(L R)}$ or $e_{C \mid A}^{(L R)}$. It is clear from Fig. 12 that, by and large, the interaction term is dominating this tendency to form disc-like structures.

In summary, the well-known tendency for $e_{A}^{(L R)} \rightarrow 1$ cannot be explained merely by the tendency for turbulence to reside preferentially in positive $\mathrm{R}_{A}$ states as this bias is only 54.6:45.4 (Table 1). However, with region 2 replacing region 1 as a contributor to such a topology as a consequence of the action of non-normality, such a ratio becomes 70:30. Each region of the $Q_{A}-R_{A}$ diagram has a novel signature, with the normal straining, $e_{B \mid A}^{(L R)}$, of greatest importance in region 5 and 6 , and of limited importance in the vortical regions. Region 1 acts counter to the general tendency for the formation of disc-like structures despite the fact that $\mathrm{R}_{A}>0$, because of the importance of the non-normal and interaction terms. In general, in this region contributions from the non-normal term are positive, while the interaction term is negative. Hence, the smaller scale anisotropies induced by viscous and non-local effects have a profound impact on the topology of flow structures in the regions where enstrophy dominates total strain. 


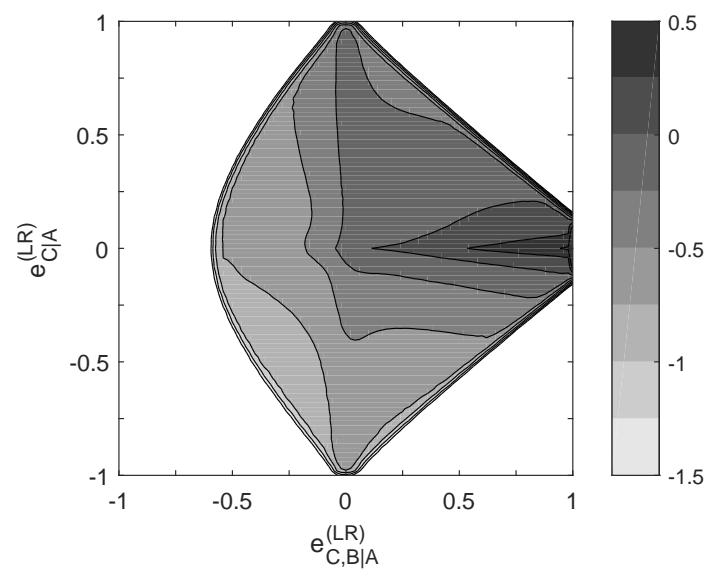

FiguRE 12. The joint distribution function for $e_{C \mid A}^{(L R)}$ and $e_{C, B \mid A}^{(L R)}$ for region 2 of the $Q_{A}-R_{A}$ diagram using the same logarithmic scaling for the contours as adopted in Fig. 10.
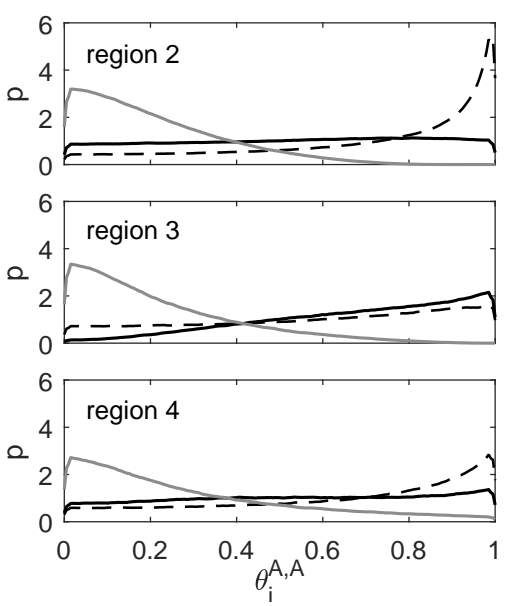
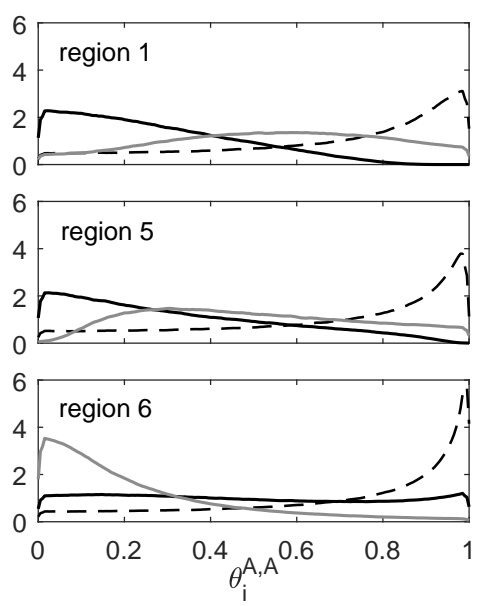

FIGURE 13. Distribution functions for the alignments, $\theta_{i}^{A, A}$ between the vorticity vector and the strain eigenvectors for $\mathrm{A}$. The black solid line is for $i=1$, the dashed line is for $i=2$ and the grey line is for $i=3$.

\section{Results: Vorticity vector and strain eigenvector alignments}

\subsection{The existing relations for $\mathrm{A}$}

The vorticity vector, $\boldsymbol{\omega}_{A}$, is typically most strongly aligned with $\mathbf{e}_{2}^{A}$ than $\mathbf{e}_{1}^{A}$ (Kerr 1985; Tsinober 2001). The probability distributions for these alignments are shown in Fig. 13. Extracting the cases where a strong alignment exists, i.e. $\theta_{i}^{A, A}>0.985\left( \pm 10^{\circ}\right)$, the results can be synthesized into the bar graphs shown in Fig. 14. This highlights the high proportion of $\theta_{i}^{A, A}>0.985$ occurrences in regions 2 and $6(\sim 36 \%$ of the total in both cases). These are also the two regions that HIT occupies preferentially relative to random, synthetic tensors with appropriate bounds on their non-normality (Keylock 2017). These relative proportions for strong alignment cases are 1.4 times and 1.2 times higher than the general occupancy proportions for these regions. Overall, $81 \%$ of cases where $\theta_{i}^{A, A}>0.985$ occur for $\theta_{2}^{A, A}$, with $16 \%$ for $\theta_{1}^{A, A}$. The propensity for alignment 

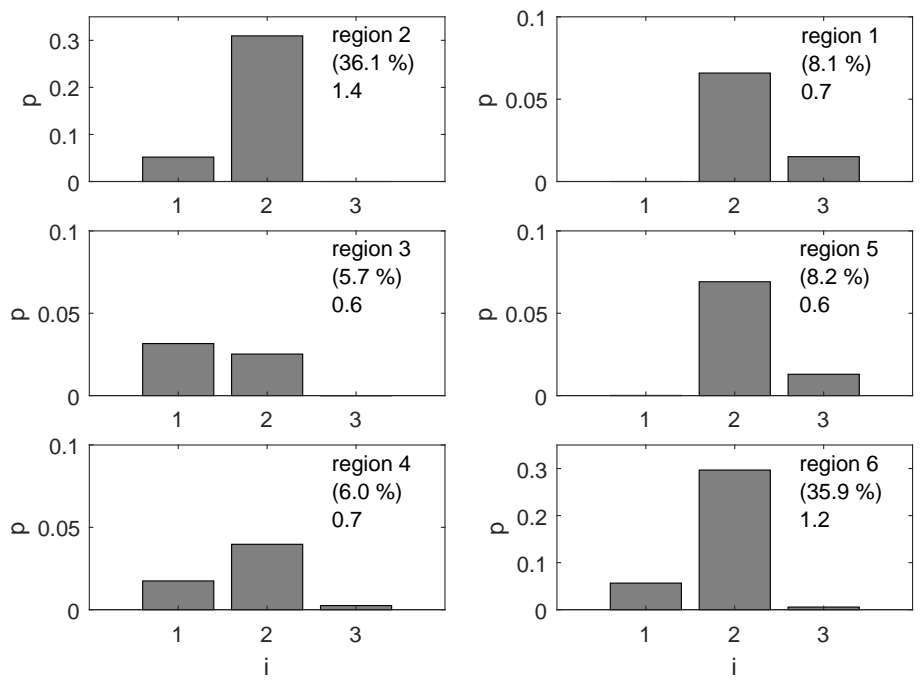

FIGURE 14. Given $\theta_{i}^{A, A}>0.985$, the bars show the probability of the alignments for particular $i$. The percentage value in brackets in each panel is the sum of the values in each panel. The number beneath this is the ratio between this percentage and that given for this region of the $\mathrm{Q}_{A}-\mathrm{R}_{A}$ diagram in Table 1.
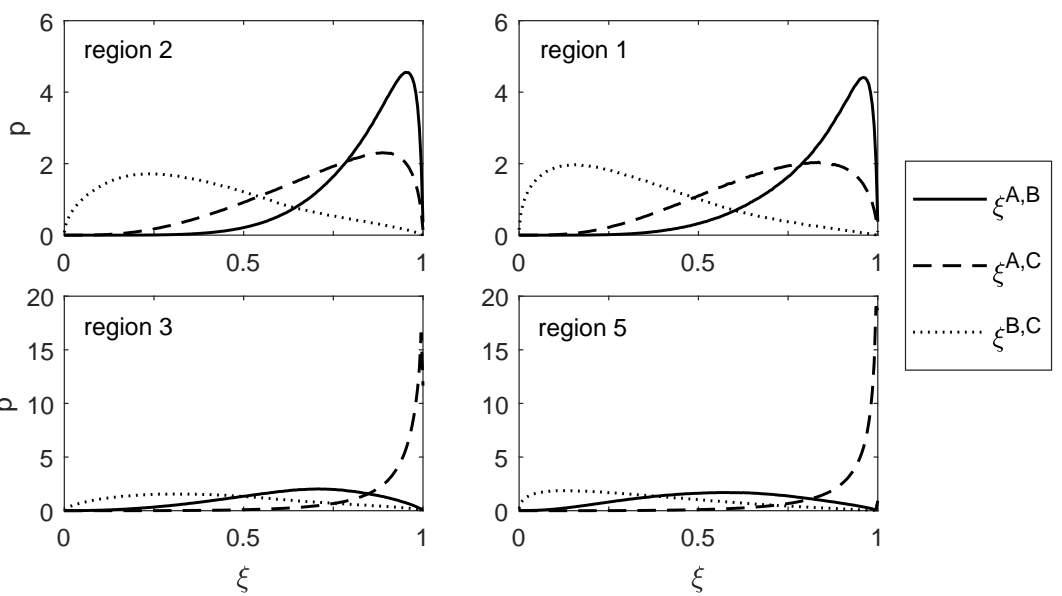

FIGURE 15. The probability of attaining different values for $\xi$ as a function of the four regions of the $\mathrm{Q}_{A}-\mathrm{R}_{A}$ diagram where $\boldsymbol{\omega}^{B}$ is non-zero

with $\theta_{1}^{A, A}$ and $\theta_{3}^{A, A}$ is dictated by the sign of $\mathrm{R}_{A}$ where $\Delta_{L}>0$ and is dominated by $\theta_{1}^{A, A}$ below the discriminant function. Region 3 is the only part of the $\mathrm{Q}_{A}-\mathrm{R}_{A}$ diagram where $\theta_{2}^{A, A}$ alignments are of secondary likelihood.

With the decomposition adopted in this study there are three vorticity vectors and nine strain eigenvectors, permitting analysis of mutual vorticity alignments (given by $\xi$ ) or strain alignments $(\phi)$, as well as the vorticity-strain alignments $(\theta)$. In the sub-sections below, the primary findings in these respects are discussed. 

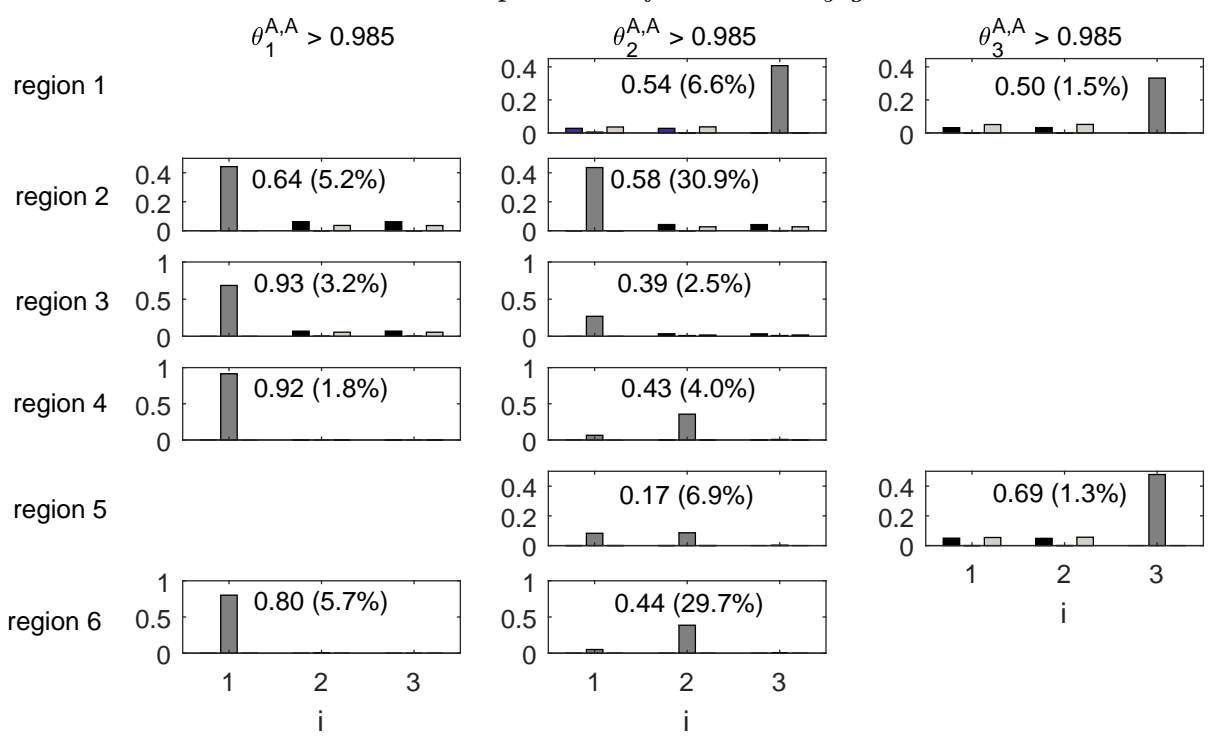

Figure 16. Given $\theta_{i}^{A, A}>0.985$, results are shown for where any $\phi_{i, j}^{B, C}>0.985$ as a function of the strain eigenvector that dictates the $\theta_{i}^{A, A}>0.985$ state. Values for $i$ are given by each group of bars, while $j \in\{1,2,3\}$ are shown in black, grey and light grey, respectively. The number in each panel is the sum of the probabilities in each panel (which does not equate to 1 because not all $\phi_{i, j}^{B, C}$ values exceed 0.985 ). The percentage in each panel indicates the fraction of the total number of cases represented by the results in a specific panel. This sums to $99.3 \%$, with the remaining $0.7 \%$ of cases occurring in the six panels that are not shown.

\subsection{Vorticity-vorticity alignments}

In order to understand the $\theta_{i}^{A, A}$ alignments discussed above, the mutual vorticity vector alignments are discussed first. Note the important result that because there is no vorticity for $B$ beneath the discriminant function then there has to be perfect alignment between the $\mathrm{A}$ and $\mathrm{C}$ vorticity vectors and $\xi^{A, C}=1$. Thus, focus is on the four regions where $\Delta_{L}>$ 0 in Fig. 15 and immediately above the discriminant function in regions 3 and 5 , there is still a strong tendency for $\xi^{A, C} \rightarrow 1$. However, when $\mathrm{Q}_{A}>0$ this is a weaker effect, with a stronger alignment for $\xi^{A, B}$ emerging. The accompanying curves for $\xi^{B, C}$ show that there is very limited strong alignment between $\boldsymbol{\omega}^{B}$ and $\boldsymbol{\omega}^{C}$, meaning there are two distinct sets of vorticity vectors in the positive $\mathrm{Q}_{A}$ regions depending on these alignments. This provides the mechanism for the observation by Tsinober et al. (1997) that points where the vorticity vector is aligned with the strain eigenvector for the maximal eigenvalue are a distinct set compared to those aligning with the intermediate eigenvector: the orientation of $\boldsymbol{\omega}^{A}$ is driven by either $\boldsymbol{\omega}^{B}$ or $\boldsymbol{\omega}^{C}$ in different locations.

\subsection{Strain-strain eigenvector alignments}

In Fig. 16, the strong strain-strain alignments, $\phi_{i, j}^{B, C}>0.985$ are shown, for the cases where strong alignments between the vorticity vector and strain eigenvectors for $A$ have already been determined (i.e. the cases highlighted in Fig. 14). Each panel potentially contains nine bars representing the three values for $j$ (black, grey, white) as a function of the three values for $i$ given by the number on the x-axis. From this diagram there is a sense of how mutual strain alignments are conditioning the observed behaviour for $A$.

The first thing to note is that not all of the eighteen possible panels are shown in this figure, as those excluded only represented $0.7 \%$ of the total cases where $\phi_{i, j}^{B, C}>0.985$ 

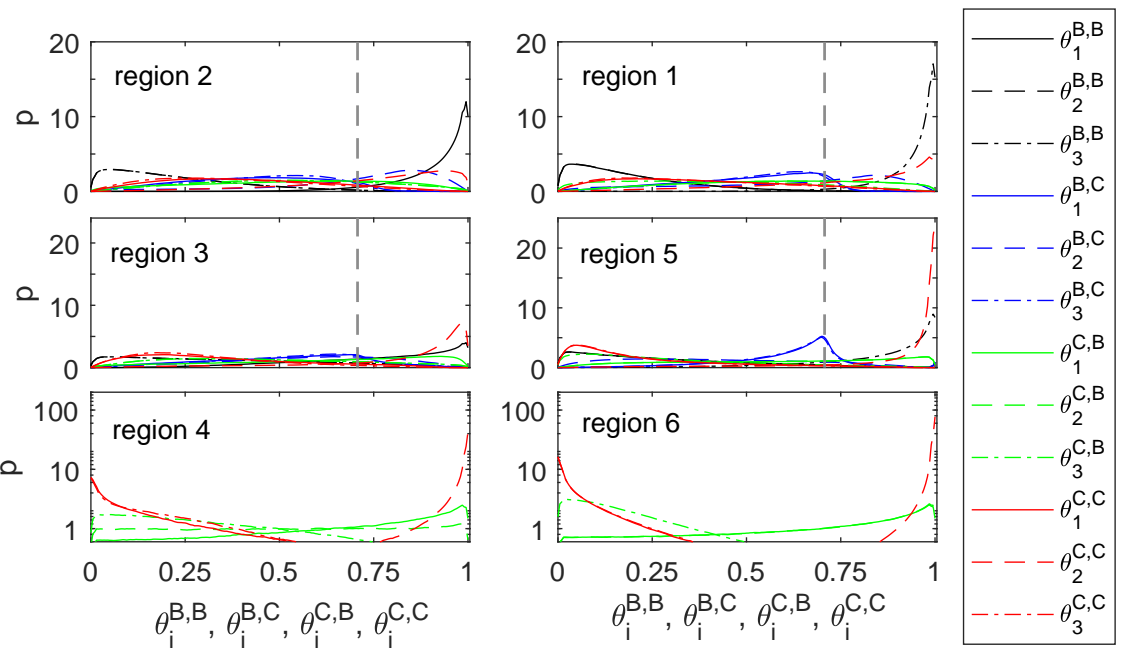

FIGURE 17. The distribution functions for the alignments, $\theta_{i}^{B, B}$ (black), $\theta_{i}^{B, C}$ (blue), $\theta_{i}^{C, B}$ (green) and $\theta_{i}^{C, C}$ (red) between the vorticity vector and the strain eigenvectors. The solid lines are for $i=1$, the dashed lines are for $i=2$ and the dot-dashed lines are for $i=3$. The vertical, dashed grey lines indicate an angle of $\pm 45^{\circ}$.

(hence, the percentages in all twelve panels shown sum to $99.3 \%$ ). Secondly, when $\theta_{1}^{A, A}>$ 0.985 (the left hand column of panels), strong strain-strain alignments are much more likely. Thus, when the vorticity vector for $A$ is aligned with the eigenvector for the leading eigenvalue, there is a $64 \%$ to $93 \%$ chance that there is a strong alignment for the strain eigenvectors for B and C. Almost exclusively, the strong alignment is for $\phi_{1,2}^{B, C}>0.985$ and these occurrences arise on the left-hand side of the $\mathrm{Q}_{A}-\mathrm{R}_{A}$ diagram (regions 2, 3,4 ) and beneath the discriminant function (regions 4 and 6 ). While $\theta_{3}^{A, A}>0.985$ only make up $2.8 \%$ of cases (right-hand column), strong strain-strain alignments are also likely ( $50 \%$ to $69 \%$ chance), are dominated by $\phi_{3,2}^{B, C}>0.985$ and occur on the right-hand side of the $\mathrm{Q}_{A}-\mathrm{R}_{A}$ diagram above the discriminant function (regions 1 and 5 ). Hence, in both the left and right columns it is the intermediate eigenvector for $C$ that dominates the strain-strain alignment.

Mutual strain-strain alignments are of reduced relative importance where $\theta_{2}^{A, A}>0.985$ ( $17 \%$ to $58 \%$ likelihood). In regions 1,2 , and 3 the relation to the left and right sides of the $\mathrm{Q}_{A}-\mathrm{R}_{A}$ diagram is preserved, with $\phi_{3,2}^{B, C}>0.985$ dominant in region 1 and $\phi_{1,2}^{B, C}>0.985$ in regions 2 and 3 . However, in regions 4,5 and 6 , the most important strain-strain alignment where $\theta_{2}^{A, A}>0.985$ is between the two intermediate eigenvectors, i.e. $\phi_{2,2}^{B, C}>0.985$.

In summary, strain-strain coupling between $B$ and $C$ is of greater importance for explaining vorticity-strain alignments for A when the alignment is not of the dominant, intermediate eigenvector type. However, irrespective of the nature of $\theta_{i}^{A, A}$, alignments are with the intermediate eigenvector of $\mathrm{C}$.

\subsection{Vorticity and strain alignments for B and C}

In addition to the mutual strain-strain and vorticity-vorticity alignments, it is also of interest to determine how the vorticity-strain alignments for $B$ and $C$ underpin the alignments for A. Figure 17 shows the probability curves for $\theta_{i}^{B, B}, \theta_{i}^{B, C}, \theta_{i}^{C, B}$, and $\theta_{i}^{C, C}$. It is noticeable that strong alignments for particular terms are more probable where 
$\mathrm{R}_{A}>0$ in regions 1,5 , and 6 . The absence of a rotation tensor for $\mathrm{B}$ beneath the discriminant function means that there are fewer alignments to evaluate in regions 4 and 6 , and the results in this region are plotted using a logarithmic ordinate because strong $\theta_{2}^{C, C}$ alignments (red, dashed line) completely dominate the statistics. The importance of this alignment persists into regions 3 and 5 , particularly in the latter case near the Vieillefosse tail. However, strong alignments for $\theta_{1}^{B, B}$ (solid, black line) and $\theta_{3}^{B, B}$ (dotdashed, black line) emerge for regions 3 and 5 , respectively, as $\boldsymbol{\omega}^{B}$ becomes non-zero, with these terms dominating the alignments in regions 1 and 2 , where $\mathrm{Q}_{A}>0$. Given the strict structure to the eigenvalues for $S_{B}$, it is necessary that these alignments are coupled to the extensive (regions 2 and 3 ) and compressive (regions 1 and 5 ) eigenvalues for the rod-like and disc-like structures, respectively. The cross-terms (green and blue lines) are of more minor importance to the alignment structure. However, where they are defined, $\theta_{1}^{B, C}$ and $\theta_{3}^{B, C}$ have maxima at $\pm 45^{\circ}$ (highlighted by the vertical, gray, dashed line), and this effect is most pronounced in region 5 . The terms that are most prone to exhibit orthogonality rather than alignment are $\theta_{3}^{C, B}$ (green, dot-dashed line) and $\theta_{1}^{C, C}$ (red, solid line). This effect is particularly true for the latter term and in regions 4 and 6. Overall, the region where one form of alignment does not really dominate is region 3 , where both $\theta_{1}^{B, B}$ and $\theta_{2}^{C, C}$ are of similar likelihood. Thus, in this region, in particular, there are two mechanisms driving the alignments, which explains why large values for $\theta_{1}^{A, A}$ and $\theta_{2}^{A, A}$ are of similar probability in Fig. 13.

\subsection{An explanation of observed alignments for $\mathrm{A}$}

Based on the above, previous observations on alignment structure may be reconsidered, focusing in particular on the intermediate $\theta_{2}^{A, A}$ alignment. Recalling from Fig. 15 that $\xi^{A, C}=1$ by definition in regions 4 and 6 , then the dominance of the $\theta_{2}^{C, C}$ term in Fig. 17 demonstrates that the non-normal tensor drives vorticity-strain alignment beneath the discriminant function. A similar explanation still applies in region 5, where $\xi^{A, C}$ and $\theta_{2}^{C, C}$ are still dominant. However, the emergence of $\theta_{3}^{A, A}$ alignments is driven by $\theta_{3}^{B, B}$ in Fig. 17. Region 3 is similar to region 5 in that $\xi^{A, C} \rightarrow 1$ and there is a strong $\theta_{2}^{C, C}$ alignment in Fig. 17, explaining the intermediate eigenvector alignment for A. However, this is the region of the $\mathrm{Q}_{A}-\mathrm{R}_{A}$ diagram where $\theta_{2}^{A, A}$ alignments are less probable than $\theta_{1}^{A, A}$, and is also the region where strong $\phi_{i, j}^{B, C}$ alignments are the most probable given strong $\theta_{1}^{A, A}$ alignments, with the dominant term $\phi_{1,2}^{B, C}$ (Fig. 16). Hence, the strong mutual alignment of the strain eigenvectors means that $\theta_{1}^{A, A}$ can emerge where the normal straining is greater in magnitude, which is not infrequent (Fig. 3).

In regions 1 and 2, the normal mutual vorticity-strain alignments are dominant and occur with the third and first eigenvectors, respectively (Fig. 17). It is also the case that $\xi^{A, B}$ is more strongly aligned than $\xi^{A, C}$, meaning that the emergence of $\theta_{2}^{A, A}$ as the dominant alignment is particularly surprising. In the context of the observation by Tsinober that this emerges because alignments with the primary eigenvector occurs on a different set of points, then Fig. 14 shows that alignments with the third eigenvector are not much less frequent when the different relative frequency of strong alignment occurrences between regions $1(8.1 \%)$ and $2(36.1 \%)$ is ignored. In either case, the kinematic properties readily explain the sub-dominant alignments with the first and third strain eigenvectors. That the intermediate eigenvector alignment dominates is then a consequence of the magnitude of the non-normal contributions to the dynamics. In particular, the strongly negative values for $\kappa_{Q}^{(S)}$ in Fig. 3 show that straining is dominated by the non-normal terms. Furthermore, the top two panels in the central column of Fig. 16 
show that when $\theta_{2}^{A, A}$ is the dominant alignment, $\phi_{3,2}^{B, C}>0.985$ or $\phi_{1,2}^{B, C}>0.985$ more frequently than elsewhere in the $\mathrm{Q}_{A}-\mathrm{R}_{A}$ diagram. With the non-normal strain then greater in magnitude, the result is $\theta_{2}^{A, A}$ dominates $\theta_{1}^{A, A}$, and $\theta_{3}^{A, A}$. Hence, the intermediate alignment detected in the early direct numerical simulations of HIT (Kerr 1985; Ashurst et al. 1987) emerges as a direct consequence of the magnitude of non-normal effects in the vortical regions - the kinematics highlight stronger relations with $B$ than $C$ in regions 1 and 2 , although elsewhere in the $\mathrm{Q}_{A}-\mathrm{R}_{A}$ diagram, non-normal effects may dominate the kinematic relations.

\section{Conclusion}

A radical interpretation of the additive decomposition of the velocity gradient tensor, A into normal and non-normal tensors, B and C, is to state that while strain occurs everywhere, no net, coherent enstrophy arises beneath the discriminant function of the $\mathrm{Q}_{A}-\mathrm{R}_{A}$ diagram. Because the eigenvalues for $\mathrm{S}_{B}$ and $\Omega_{B}$ are the real and imaginary parts of the eigenvalues for $A$, respectively, the latter does not exist in regions 4 and 6 , and any observed vorticity comes from the non-normal contribution. However, because $\left\|\Omega_{C}\right\|^{2}=\left\|S_{C}\right\|^{2}$, this is counter-balanced by a term contributing to the straining.

It was something of a surprise to fluid mechanics when the first direct numerical simulations of HIT showed a preferential alignment between the vorticity vector and the eigenvector for the second eigenvalue of the strain tensor rather than the first eigenvalue (Kerr 1985; Ashurst et al. 1987). This study has shown the critical role of non-normality in explaining this phenomenon. In regions 4 and 6 there is a perfect alignment between the vorticity vectors for $\mathrm{A}$ and $\mathrm{C}$, i.e. $\xi^{A, C}=1$, and this term still dominates in regions 3 and 5. Coupled to the tendency for the vorticity vector for $\mathrm{C}$ to align with the eigenvector for the intermediate strain eigenvalue for C, i.e. $\theta_{2}^{C, C}$, this explains why $\theta_{2}^{A, A}$ is prevalent in these regions. In regions 1 and 2 , where enstrophy dominates strain, the normal terms dominate the kinematic relations, which imply alignments with the first and third eigenvectors. However, the weak normal straining compared to the magnitude of the non-normal straining term ensures that dynamically, the $\theta_{2}^{A, A}$ term still emerges as dominant, given the strong strain-strain alignments between $\phi_{3,2}^{B, C}$ (region 1) and $\phi_{1,2}^{B, C}$ (region 2), as seen in Fig. 16.

In addition to the preference for $\theta_{2}^{A, A}$ to be the dominant alignment in HIT, we have also investigated the well-known property for the strain eigenvalues for turbulence to tend towards disc-like rather than rod-like structures. Simply considering the strain eigenvalues, then it should be regions 1,5 and 6 on the right-hand side of the $\mathrm{Q}_{A}-\mathrm{R}_{A}$ diagram that drive the tendency for the formation of disc-like structures, and homogeneous, isotropic turbulence is in these three regions on about $55 \%$ of occasions. However, the teardrop shape of the $\mathrm{Q}_{A}-\mathrm{R}_{A}$ diagram means that region 2 is occupied much more frequently than region 1 in the ratio 26.5:11.1. Non-normal straining effects are more important in the vortical regions, and there are two terms that arise: the non-normal production term occurs preferentially in region 1 and is approximately uniformly distributed, with no preference to drive the formation of rod- or disc-like structures. On the other hand, in region 2, the interaction term is dominant and this is strongly biased towards the creation of disc-like structures. As a consequence, even though the normal straining in region 2 is driving a tendency to form rod-like structures, this weak term is over-ridden by the magnitude of the interaction term. Thus, it is regions 2,5 and 6 that drive the tendency to form disc-like structures, and these are expressed on $70 \%$ of occurrences, leading to the dominance of disc-like over rod-like straining structure. 
Our results highlight the difficulty of applying eigenvalue-focused thinking to the dynamics of complex systems such a turbulence. This is now well-known in hydrodynamic stability where transient effects due to non-normality, characterized by the pseudospectra, explain the timescales for instabilities to emerge (Reddy et al. 1993; Trefethen et al. 1993; Trefethen \& Embree 2005). The results of this study, summarized above, show how important non-normal effects are for the dynamics, and this is particularly the case for the alignment of the vorticity vector with the intermediate strain eigenvector, as explained above. The advantage of the Schur transform in this respect is that the non-normality, $\mathrm{N}$ is projected into the Schur matrix, $\mathrm{T}$, where it can be treated in a similar way to the eigenvalues, $\Lambda$. Hence, this permitted the definition of $\mathrm{B}=\mathrm{U} \wedge \mathrm{U}^{*}$ and $\mathrm{C}=\mathrm{UNU}^{*}$ in this paper. In contrast, the eigenvalue decomposition projects the non-normality into the departure of the eigenvectors from a unitary form, making it harder to directly compare normal (local) and non-normal (non-local) effects.

It is only really along the Vieillefosse tail that normal effects, in particular, normal straining, dominate the dynamics. This helps explain the success of the restricted Euler model (Cantwell 1992) in approximating the dynamics of the VGT, but the more complex behaviour in regions 1, 2 and 3, in particular, demonstrates the difficulty of understanding the non-local effects that predominate without considering both the eigenvalues and the non-normal contributions. Regarding the latter, of particular importance is the non-normal contribution to the balance between enstrophy and strain, $\left\|S_{C}\right\|$, and the non-normal production and interaction production terms defined in Table 2, all of which may be obtained from the equation for the evolution of strain and rotation in eq. (2.14). The decomposition introduced here also permits metrics for turbulence behaviour to be reconsidered. The Lund and Rogers normalization of the strain eigenvalues may be expanded into constituent terms as shown in eq. (2.24), while the existence of vorticity vectors and strain eigenvectors for $A, B$, and $C$ permits alignment properties to be examined in a new way as defined in equations (2.25) to (2.27). These terms and metrics that can serve as a basis for evaluting existing models for the dynamics of the VGT (Wilczek \& Meneveau 2014; Johnson \& Meneveau 2016), and for formulating new models of this type.

\section{Appendix A. Proof of the existence of a Schur transform}

A Schur transform (Schur 1909) always exists for a $n \times n$ matrix, A

$$
\mathrm{A}=\mathrm{UTU}^{*}
$$

and this decomposition can be arrived at by induction on $n$ because the Schur transform implies a set of nested subspaces that are $A$ invariant. Given that the columns of $U$ form an orthonormal basis, for $n=1, \mathrm{U}=1$. For $n>1$, if $\lambda_{1}$ is an eigenvalue of $\mathrm{A}$ and then one may denote the set of all eigenvectors for $\lambda$ as $W$ with orthogonal complement, $W^{\perp}$. One may then choose arbitrary orthonormal bases $Y_{1}$ and $Y_{2}$ that span $W$ and $W^{\perp}$, such that the following submatrices are obtained

$$
\left[\begin{array}{ll}
Y_{1} & Y_{2}
\end{array}\right]^{*} A\left[\begin{array}{ll}
Y_{1} & Y_{2}
\end{array}\right]=\left[\begin{array}{cc}
\lambda_{1} & A_{12} \\
0 & A_{22}
\end{array}\right]
$$

The right-hand side yields the desired upper-triangular form for $\mathrm{T}$ apart from the submatrix $A_{22}$. Hence, using the hierarchical subspace concept and redefining the above subspaces with respect to $\mathrm{A}_{22}$ initially, and continuing with such operations $n-1$ times results in an upper triangular matrix that may be decomposed into a diagonal matrix of eigenvalues and the non-normality matrix, $\mathrm{T}=\mathrm{L}+\mathrm{N}$, as desired. 
The Schur transform (Schur 1909) may be implemented in a complex or a real form, where the latter is only quasi-upper triangular if the eigenvalues are complex, with the conjugate pair forming a $2 \times 2$ Jordan block. Hence, the complex form is adopted throughout this paper to avoid this eventuality.

This research was supported by a Royal Academy of Engineering/Leverhulme Trust Senior Research Fellowship LTSRF1516-12-89 awarded to the author. He is grateful to Charles Meneveau at Johns Hopkins University, as well as Christos Vassilicos and colleagues at the Department of Aeronautics, Imperial College London, for their hospitality while completing this research.

\section{REFERENCES}

Ashurst, W. T., Kerstein, A. R., Kerr, R. A. \& Gibson, C. H. 1987 Alignment of vorticity and scalar gradient with strain rate in simulated Navier-Stokes turbulence. Phys. Fluids 30, 2343-2353.

Betchov, R. 1956 An inequality concerning the production of vorticity in isotropic turbulence. J. Fluid Mech. 1, 497-504.

Biferale, L., Chevillard, L., Meneveau, C. \& Toschi, F. 2007 Multiscale model of gradient evolution in turbulent flows. Phys. Rev. Lett. 98, 214501.

CAntwell, B. J. 1992 Exact solution of a restricted Euler equation for the velocity gradient tensor. Phys. Fluids A 4 (4), 782-793.

Cantwell, B. J. 1993 On the behavior of velocity gradient tensor invariants in direct numerical simulations of turbulence. Phys. Fluids A 5, 2008-2013.

Chong, M. S., Perry, A. E. \& Cantwell, B. J. 1990 A general classification of threedimensional flow fields. Phys. Fluids A 2, 765-777.

Dubief, Y. \& Delcayre, F. 2000 On coherent-vortex identification in turbulence. J. Turbul. 1 (011).

Eberlein, P. J. 1965 On measures of non-normality for matrices. Amer. Math. Mon. 72, 995996.

Elsinga, G. E. \& Marusic, I. 2010 Evolution and lifetimes of flow topology in a turbulent boundary layer. Phys. Fluids 22, 015102.

Girimaji, S. S. \& Pope, S. B. 1990 A diffusion model for velocity gradients in turbulence. Phys. Fluids 2 (2), 242-256.

Golub, G. H. \& van LoAn, C. F. 2013 Matrix Computations, 4th edn. Johns Hopkins University Press.

Goto, S. 2008 A physical mechanism of the energy cascade in homogeneous isotropic turbulence. J. Fluid Mech. 605, 355-366.

HenRICI, P. 1962 Bounds for iterates, inverses, spectral variation and fields of values of nonnormal matrices. Numer. Math. 4, 24-40.

Higham, J. E., Brevis, W. \& Keylock, C. J. 2016 A rapid non-iterative proper orthogonal decomposition based outlier detection and correction for PIV data. Meas. Sci. Tech. 27, 125303.

Hunt, J. C. R., Wray, A. A. \& Moin, P. 1988 Eddies, stream, and convergence zones in turbulent flows. Tech. Rep. CTR-S88. Center for Turbulence Research, Stanford University.

JeOnG, E. \& GiRIMAJI, S. S. 2003 Velocity-gradient dynamics in turbulence: effect of viscosity and forcing. Theoret. Comput. Fluid Dyn. 16, 421-432.

Jimenez, J. 1992 Kinematic alignmernt effects in turbulent flows. Phys. Fluids A 4, 652-654.

Johnson, P. L. \& Meneveau, C. 2016 A closure for Lagrangian velocity gradient evolution in turbulence using recent-deformation mapping of initially Gaussian fields. J. Fluid Mech. $\mathbf{8 0 4}, 387-419$.

KERR, R. M. 1985 Higher-order derivative correlations and the alignment of small-scale structures in isotropic, numerical turbulence. J. Fluid Mech. 153, 31-58.

KEYLOCK, C. J. 2017 Synthetic velocity gradient tensors and the identification of statistically significant aspects of the structure of turbulence. Phys. Rev. Fluids 2 (004600). 
Kuo, A. Y.-S. \& Corrsin, S. 1972 Experiment on the geometry of the fine-structure regions in fully turbulent fluid. J. Fluid Mech. 56, 447-479.

Lawson, J. M. \& Dawson, J. R. 2015 On velocity gradient dynamics and turbulent structure. J. Fluid Mech. 780, 60-98.

LEE, S. L. 1995 A practical upper bound for departure from normality. SIAM J. Matrix Analys. Appl. 16, 462-468.

LI, Y. \& Meneveau, C. 2007 Material deformation in a restricted Euler model for turbulent flows: analytic solution and numerical tests. Phys. Fluids 19, 015104.

Li, Y., Perlman, E., Wan, M., Yang, Y., Burns, R., Meneveau, C., Chen, S., Szalay, A. \& EYINK, G. 2008 A public turbulence database cluster and applications to study Lagrangian evolution of velocity increments in turbulence. J. Turbulence 9 (31).

Lund, T. S. \& Rogers, M. M. 1994 An improved measure of strain state probability in turbulent flows. Phys. Fluids 6 (5), 1838-1847.

Lundgren, T. S. 1982 Strained spiral vortex model for turbulent structures. Phys. Fluids 25, 2193-2203.

Lüthi, B., Holzner, M. \& Tsinober, A. 2009 Expanding the Q-R space to three dimensions. J. Fluid Mech. 641, 497-507.

Martin, J., Dopazo, C. \& Valiño, L. 1998 Dynamics of velocity gradient invariants in turbulence: restricted Euler and linear diffusion models. Phys. Fluids 10, 2012-25.

Meneveau, C. 2011 Lagrangian dynamics and models of the velocity gradient tensor in turbulent flows. Ann. Rev. Fluid Mech. 43, 219-245.

Nomura, K. K. \& Post, G. K. 1998 The structure and dynamics of vorticity and rate of strain in incompressible homogeneous turbulence. J. Fluid Mech. 377, 65-97.

Ohkitani, K. \& Kishiba, S. 1995 Nonlocal nature of vortex stretching in an inviscid fluid. Phys. Fluids 7 (2), 411-421.

Paul, I., PApadakis, G. \& VAssilicos, J. C. 2017 Genesis and evolution of velocity gradients in a spatially developing turbulence. J. Fluid Mech. 815, 295-332.

Perry, A. E. \& Chong, M. S. 1987 Description of eddying motions and flow patterns using critical-point concepts. Annu. Rev. Fluid Mech. 19, 125-155.

Reddy, S. C., Schmid, P. J. \& Henningson, D. S. 1993 Pseudospectra of the Orr-Sommerfeld operator. SIAM J. Appl. Math. 53, 15-47.

SchuR, I. 1909 Über die charakteristischen Wurzeln einer linearen Substitution mit einer Anwendung auf die Theorie der Integralgleichungen. Math. Ann. 66, 488-510.

Taylor, G. I. 1938 The spectrum of turbulence. Proc. R. Soc. Lond. A 164, 476-490.

Trefethen, L. N. \& Embree, M. 2005 Spectra and Pseudospectra: The Behavior of Nonnormal Matrices and Operators. Princeton University Press.

Trefethen, L. N., Trefethen, A. E., Reddy, S. C. \& Driscoll, T. A. 1993 Hydrodynamic stability without eigenvalues. Science 261, 578-584.

Tsinober, A. 2001 Vortex stretching versus production of strain/dissipation. In Turbulence Structure and Vortex Dynamics (ed. J. C. R. Hunt \& J. C. Vassilicos), pp. 164-191. Cambridge University Press.

Tsinober, A., Shtilman, L. \& Vaisburd, H. 1997 A study of properties of vortex stretching and enstrophy generation in numerical and laboratory turbulence. Fluid Dyn. Res. 21, 477-494.

Vieillefosse, P. 1984 Internal motion of a small element of fluid in an inviscid flow. Physica A 125, 150-162.

Wan, M., Chen, S., Eyink, G., Meneveau, C., Perlman, E., Burns, R., Li, Y., SzaLAY, A. \& Hamilton, S. 2016 Johns Hopkins Turbulence Database (JHTDB). http: //turbulence.pha.jhu.edu/datasets.aspx.

Wan, M., Xiao, Z., Meneveau, C., Eyink, G. L. \& Chen, S. 2010 Dissipation-energy flux correlations as evidence for the Lagrangian energy cascade in turbulence. Phys. Fluids $22(6), 1-4$.

Wilczek, M. \& Meneveau, C. 2014 Pressure Hessian and viscous contributions to velocity gradient statistics based on Gaussian random fields. J. Fluid Mech. 756, 191-225. 